\title{
Non-equilibrium effects of core-cooling and time-dependent internal heating on mantle flush events
}

\author{
D. A. Yuen' ${ }^{1}$, S. Balachandar ${ }^{2}$, V. C. Steinbach ${ }^{3}$, S. Honda ${ }^{4}$, D. M. Reuteler', J. J. Smedsmo ${ }^{1}$ and G. S. Lauer
}

${ }^{2}$ Dept. of Geology, Geophysics and Minnesota Supercomputer Inst., Univ. of Minnesota, Minneapolis, MN 55415-1227, USA

${ }^{2}$ Dept. of Theoretical and Applied Mechanics, University of Illinois, Urbana, IL 61801, USA

${ }^{3}$ Inst. für Geophysik und Meteorologie, Universität zu Köln, 50923 Köln, Germany

${ }^{4}$ Dept. of Earth and Planetary Systems Science, Faculty of Science, University of Hiroshima, Higashi-Hiroshima 739, Japan

Received 10 October 1994 - Accepted 7 June 1995 - Communicated by D. Sornette

Abstract. We have examined the non-cquilibrium effects of core-cooling and time-dependent internal-heating on the thermal evolution of the Earth's mantle and on mantle flush events caused by the two major phase transitions. Both two- and three-dinnensional models have been employed. The mantle viscosity responds to the secular cooling through changes in the averaged temperature field. A viscosity which decreases algebraically with the average temperature has been considered. The time-dependent internal-heating is prescribed to decrease exponentially with a single decay time. We have studied the thermal histories with initial Rayleigh numbers between $2 \times 10^{7}$ and $10^{8}$. Flush events, driven by the non-equilibrium forcings, are much more dramatic than those produced by the equilibrium boundary conditions and constant internal heating. Multiple flush events are found under non-equilibrium conditions in which there is very little internal heating or very fast decay rates of internal-heating. Otherwise, the flush events take place in a relatively continuous fashion. Prior to massive flush events small-scale percolative structures appear in the 3D temperature fields. Time-dependent signatures, such as the surface heat flux, also exhibits high frequency oscillatory patterns prior to massive flush events. These two observations suggest that the flush event may be a self-organized critical phenomenon. The Nusselt number as a function of the time-varying $\mathrm{Ra}$ does not follow the Nusselt vs. Rayleigh number power-law relationship based on equilibrium (constant temperature) boundary conditions. Instead $N u(t)$ may vary non-monotonically with time because of the mantle flush events. Convective processes in the mantle operate quite differently under non-equilibrium conditions from its behavior under the usual equilibrium situations.

\section{Introduction}

In the last four years numerical simulations of mantle convection have revealed a new type of instability from the presence of the endothermic phase transition at 670 $\mathrm{km}$ depth (Machetel and Weber, 1991; Honda et al., 1993a and b; Tackley et al., 1993, 1994; Steinbach et al., 1993; Weinstein, 1993; Honda and Yuen, 1994; Ita and King, 1994 and Yuen et al, 1994a). These simulations have raised the possibility that the present state of the mantle is partially layered, with the degree of stratification varying both in space and time. These issues are especially relevant to one of the most important question in the solid Earth geosciences of whether the mantle convects as a single layer or the circulation is layered. There are also accumulating evidences from seismic tomography (van der Hilst et al., 1991; Fukao et al., 1992; Ohtaki and Kaneshima, 1994; van der Hilst, 1995) which show both slabs lying horizontally in the transition zone and some of the slabs going through the 670 $\mathrm{km}$ discontinuity. Characteristic eigen-decomposition of the tomographic data has also revealed the possibility of partial layering of the present day mantle (Balachandar 1995). The periodic discharge of cold material accumulated in the transition zone has been called mantle "flush" instability. Most of the work on this subject has been based on the assumption of constant core-mantle boundary (CMB) temperature (e.g. Machetel and Weber, 1991; Honda et al., 1993a; Tackley et al., 1993; Weinstein, 1993) and constant rate of internal-heating (Zhao et al., 1992; Weinstein, 1993; Solheim and Peltier, 1994). But these assumptions are not valid over a long time scale of the order of a few billion years, because of the cooling of the core by the mantle circulation. Moreover, the major radioactive elements in the mantle have a mean half-life of between $1 / 4$ to around two times the age of the present Earth. These two timedependent forcings of the mantle convective system, lead to non-equilibrium situations in which the time-varying 
thermal coupling between the core and the mantle and the time-dependent nature of the internal heating would cause the mantle circulation to respond in some unusual fashion. These aspects are at the heart of the phenomena of self-organization and dissipative structures (e.g. Nicolis and Prigogine, 1977; Haken, 1983), which have been studied by physicists for many years and are now being appreciated by the geoscientists (e.g. Ortoleva, 1994).

This problem of strong interaction between two systems (here the core and the mantle) is common in many areas of science. Thus far it has not received much attention in the area of mantle convection. Only a few investigations (Steinbach et al., 1993; Nakakuki, 1993; Arkani-Hamed, 1994; Honda and Yuen, 1994) have emphasized the thermal evolution of the mantle for cases with and without the mantle phase transitions in the presence of core-coupling. With the presence of phase transition, which offers the possibility of a threshold phenomenon (e.g. Stauffer and Aharony, 1992) like the mantle flush events, mantle convection exhibits interesting phenomena such as self-organized criticality (e.g. Bak et al., 1988), which would be of great interest to nonlinear dynamicists.

In this paper we will focus on the new understanding brought about by the non-cquilibrium aspects on mantle flush events. We are especially interested in the different responses of the mantle convection to time dependent external forcings. We will use results obtained from both two- and three-dimensional numerical simulations. First, we present results for the equilibrium situations, which will be followed by two-dimensional non-equilibrium simulations with examples drawn from the time-dependent forcing of the core-cooling boundary condition at the CMB. We then show the differences in the style of 3-D flush events between the corecooling forcing and that due to both core-cooling and time-dependent internal-heating. This will be followed by a 2-D study of the influences of different decay rates of internal-heating on the subsequent thermal evolution of the flush events. Finally we present the concluding remarks.

\section{Model Description}

We have employed a Cartesian model and Fig. 1 shows schematically the two major phase transitions and the thermal coupling of the core to the mantle at the coremantle boundary $(z=0)$. The depth of the model mantle has been set to $2000 \mathrm{~km}$ for computational reasons (Honda et al., 1993a; Steinbach and Yuen, 1994a; Honda and Yuen, 1994). The two major phase transitions, olivine to spinel and spinel to perovskite, are located at respectively 400 and $670 \mathrm{~km}$ depth. Although there may be compositional stratification across the 670 $\mathrm{km}$ boundary (Richter and McKenzie, 1981), we will

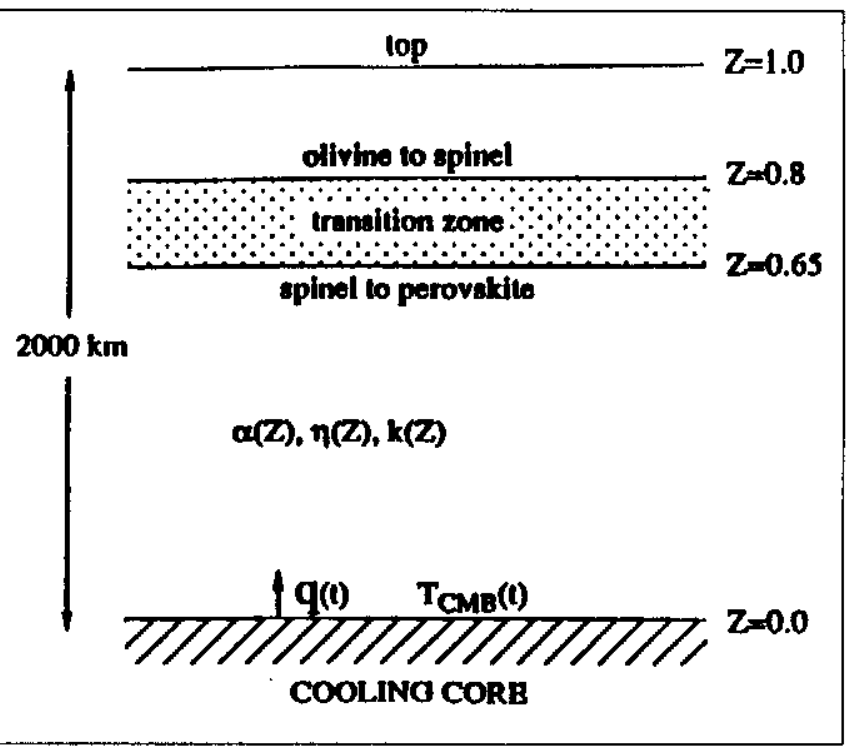

Fig. 1. Schematic diagram of the different depth ranges in the numerical model. The olivine to spinel and spinel to perovskite phase changes are located at non-dimensional depths of 0.8 and 0.65 respectively, for a model mantle of $2000 \mathrm{~km}$ depth. Thermal expansivity, thermal conductivity and viscosity are functions of depth. At each time step, the heat flux from core $q(t)$ is calculated and used in computing the new core temperalure $T_{c m b}(l)$ and the temperature drop across the mantle through an ordinary differential equation (eqn (2)). A dimensionless timestep of 0.001 corresponds to $127 \mathrm{Myr}$.

assume that this internal boundary layer is due to an endothermic phase transition (Christensen and Yuen, 1984). The buoyancy effect and the latent heat release (or absorption) at the phase transitions are modelled with an effective depth-dependent thermal expansivity (Christensen and Yuen, 1985; Steinbach and Yuen, 1992, 1994a). With this approximation the thermal expansivity consists of two parts, one describing the depthdependent background variation, prescribed based on laboratory experiments (Chopelas and Boehler, 1992) and a second part accounting for the sharp variations in the effective thermal expansivity across the phase transition zones of width 40 to $50 \mathrm{~km}$. The total thermal expansivity, as a function of $z$, is given by

$\alpha(z)=\alpha_{b}(z)+\alpha_{1}(z)+\alpha_{2}(z)$

where $\alpha_{b}(z)$ represents the background depth dependence (Balachandar and Yuen, 1994; Steinbach and Yuen, $1994 \mathrm{a})$, the localized functions $\alpha_{1}(z)$ and $\alpha_{2}(z)$ represent respectively the olivine to spinel $(z=0.80)$ and the spinel to perovskite $(z=0.65)$ phase transitions. A description of these localized functions can be found in Honda et al. (1993b) and Reuteler et al. (1994) for the 3-D cases and Steinbach and Yuen (1994a) and Honda and Yuen (1994) for the 2-D cases.

The details of the equilibrium and non-equilibrium models for mantle flush events can be found respectively in Honda et al. (1993b) and Steinbach and Yuen 
(1994a). Here we will only present a brief description of the salient features of the non-equilibrium model.

The basic equations used are the conservation of mass, momentum and energy. The governing equations are non-dimensionalized by the thermal diffusion time across the layer, based on the surface value of the thermal diffusivity, as the time scale; $\Delta T_{0}$, the initial temperature difference between the surface and the CMB, as the temperature scale; and the depth of the mantle as the length scale. Viscosity, thermal conductivity and thermal coefficient of expansion have been non-dimensionalized by their respective values at the top surface. 'T'he governing equations are simplified by taking the limit of infinite Prandtl number appropriate for mantle convection. The non-dimensionalized cquations are written within the framework of the extended-Boussinesq approximation (Christensen and Yuen, 1985 and Steinbach et al. 1989) with depth-dependent variation in viscosity, thermal expansivity and thermal expansion coefficient. Both viscous and adiabatic heatings are included in the energy equation, as well as latent heat release or consumption. A detailed comparison of the role played by depthdepcndent mantle properties on nush events caused by phase transitions can be found in Stcinbach and Yuen (1994a). The governing equations are cast in terms of the primitive variables: the velocity field, $u$, the temperature field, $T$, and the dynamical pressure, $p$. 'The equations can be found in Honda et al. (1993a) and Reuteler et al. (1993) and will not he repeated here.

The 3-D simulations will employ a Fourier-Chebyshev spectral-transform method (Balachandar and Yuen, 1994) with periodic boundary conditions along the horizontal directions. A Chebyshev expansion is used in the vertical direction and grid stretching along this direction is used to provide improved vertical resolution near the phase transition zone (Bayliss and Turkel, 1992). Stress-free boundary conditions are employed at the top and bottom impermeable boundaries. The two control variables of this model are the surface Raylcigh number, $R a_{s}$, and the surface dissipation number, $D_{s}$, which measure respectively the strength of convection and the scale height of the adiabatic temperature. $R a_{s}$ is given in terms of the initial temperature difference across the Inantle and the surface values of the thermal expansivity, viscosity and thermal diffusivity. $D_{s}$ is similarly given by the surface values of the thermal expansivity and the specific heat. The surface dissipation number and the non-dimensional adiabatic temperature evaluated at the surface are chosen to be $D_{s}=0.4$ and $T_{a}(z=1)=0.2$, respectively. The depth of the mantle in all cases is taken to be $2000 \mathrm{~km}$. Three thousand degrees have been used as the temperature difference across the mantle for the constant CMB temperature model and also for the initial temperature difference for the core-cooling models. The surface value of the thermal diffusivity is set to $10^{-6} \mathrm{~m}^{2} / \mathrm{s}$ and the surface value of the thermal expansivity to $3 \times 10^{-5} \mathrm{~K}^{-1}$. The surface viscosity can then be determined from the Rayleigh number and the other physical parameter values.

In all 3-D calculations the viscosity increases exponentially with depth and the viscosity contrast across the mantle is taken to be 10 . Thermal expansivity decreases by a factor of 4 and the thermal conductivity increases by a factor of 2.5. The spatial resolution of the spectral codc can be judged by an examination of the spectral decay of the temperature and velocity spectras. For all cases studied here we obtained a decay of the energy spectrum over several orders in magnitude, indicating adequate spatial resolution in all three directions.

An isothermal boundary condition is maintained at the top. We apply the conservation of energy for the core and arrive at an ordinary differential equation for the instantaneous temperature difference between the core and the surface, $\Delta T(t)$, (Steinbach and Yuen, 1994a), which is

$\frac{d \Delta T^{\prime}(t)}{d t}=-K N u_{b}(t) \Delta T(t)$

where $K$ is a constant, around 2, (Steinbach and Yuen, 1994a) and $N u_{b}$ is the Nusselt number evaluated at the CMB $(z=0)$. In obtaining the above formula, we have neglected the release of latent heat and gravitational potential energy from the freezing of the inner core (Stevenson et al., 1983). Owing to the exchange of heat between the core and the mantle, the temperature at the CMB decreases over time and thus decreasing the temperature difference across the mantle as given by equation (2). The resulting non-equilibrium effect is further enhanced by a temperature-dependent viscosity, as the increase in mantle viscosity from secular cooling could be as large as three to four orders in magnitude over the course of the geological time scale (Sharpe and Peltier, 1978). This dependence of effective viscosity, $\nu_{\text {eff }}(t)$, on the averaged tcmpcrature is of the form

$\nu_{e f f}=\frac{\nu_{0}}{\langle T\rangle^{m}}$

where $v_{0}$ is the initial surface viscosity, $m$, the powerlaw exponent is taken here to be an integer of value 30 (Davies, 1980) and the angle brackets indicate a volume average. This type of temperature dependence facilitates considerably the speed of the numerical computations than using a viscosity law with a local dependence on the temperature. This effective viscosity is then substituted along with the time-dependent temperalure drop across the mantle given by Eq. (2) to obtain an effective Rayleigh number, $R a_{e}$. The effective Rayleigh number at time $t$ can then be written as

$R a_{\varepsilon}(t)=R a_{s} \frac{\Delta(t)}{\Delta T(0)} \frac{<T(t)>^{m}}{<T(0)>^{m}}$

where $R a_{s}$ is the initial Rayleigh number at the surface. $R a_{e}(t)$ is then employed directly in the buoyancy term 
of the momentum cquation. 'Thus, as the core cools down, the driving force for convection would decrease with time.

Another important source of non-equilibrium forcing in mantle convection is the time-dependent nature of radioactive heating. 'The half-lives of the important radioactive elements in the mantle span from between $1 / 5$ to about 2 times the lifetime of the present-day Earth (e.g. Turcotte and Schubert, 1982). We will include the effect of time-dependent internal heating in the energy equation through a time-dependent inhomogeneous term of the form

$$
R(t)=R_{0} \exp (-\lambda t)
$$

where $R_{0}$ is the initial strength of the internal heating and $\lambda$ is the mean inverse decay time. We have considered here only one type of radioactive decay and not a whole suite of decay rates for different radioactive elements. The value of $R_{0}$ will be taken to be 20 , as compared with present-day chondritic heating strength lying between 10 and 15 (Leitch and Yuen, 1989) and values of $\lambda$ spanning from 75 to 300 will be considered. The parameter $R$ is scaled by $\frac{H d^{2}}{k \Delta T}$ where $H$ is the radioactive heat generation per unit mass, $d$ is the depth of the mantle, $k$ is the surface thermal conductivity and $\Delta T$ is the temperature difference across the mantle. For time-dependent heating situations the quantily $R$ varies with time.

Apart from the 3-D model, two 2-D models are also employed and they both are based on Cartesian geometry and employ the stream function, vorticity and temperature formulation. The first is based on a finitedifference algorithm (Honda and Yuen, 1989) and the second is based on a finite-element methodology with streamwise upwind Petrov Galerkin scheme (Hansen and Ebel, 1988). Periodic horizontal boundary conditions are employed in the finite-difference code, whilc reflecting side wall boundary conditions are used in the finiteelement code.

\section{Results}

We have taken the density changes of $8 \%$ and $10 \%$ respectively for the olivine to spinel and the spinel to perovskite phase changes. The Clapeyron slopes for the olivine to spinel and the spinel to perovskite transitions are specified to be respectively 3 and $-3 \mathrm{MPa} / \mathrm{K}$ (Katsura and Ito, 1989; Chopelas, 1991; Ito and Takahashi, 1989).

\subsection{Equilibrium results}

All of the existing three-dimensional investigations of flush events (Honda et. al., 1993 a and b; Tackley et al., 1993 and 1994; Yuen et al. 1994a and Reuteler et al., 1994) have bcen conducted under equilibrium boundary condition (constant CMB temperature). In this section we will review the features of the three-dimensional perspective shots of the temperature fields for two vastly different Rayleigh numbers because of its implications on the transition from layered to unlayered state and also on percolative structures. There is no internal heating for these two cases whose surface Rayleigh numbers differ by a factor of 50 . Figure 2 shows the evolution of $R a_{s}=2 \times 10^{6}$, a case taken from Honda et al. (1993b) and Fig. 3 shows the snapshots of $R a_{s}=10^{8}$, a case taken from Yuen et al. (1994a). The simulation at the lower $R a_{s}$ was started from a case without any phase transitions (Balachandar et al., 1992), while the solution for $R a_{s}=10^{8}$ was started from a well-developed solution with two phase transitions at $R a_{s}=4 \times 10^{7}$. The lower Rayleigh number solution has a grid-like structure consisting of cold descending sheets and a few plumes at one corner. Starting from the left column and going over to the right, we observe the flushing events, as cold material hits the bottom and leaves tell-tale white tracks at the bottom. In contrast, at $R a_{s}=10^{8}$, flushing events no longer can take place because of the much less residence time in the transition zone and the development of hard-turbulent convection in the upper-mantle leading to percolative, fractal-like structures of the cold material. There are many more plumes in the lower mantle but almost all of them are trapped there and not able to penetrate into the upper-mantle as in the case of the lower Rayleigh number. This propensity for greater amount of layering at higher Rayleigh number was already shown in the 2-D solutions (Christensen and Yuen, 1985; Zhao et al., 1992; Steinbach et al., 1993), which also spanned two orders in the magnitude of $R a_{s}$. A comparison of the patterns of the cold material between these two very different Rayleigh numbers reveals a distinct transition in the morphology. In the one case $\left(R a_{s}=2 \times 10^{6}\right)$, an organized grid is prevalent, while the structure of the high $R a_{s}$ case reminds one of condensation process of collecting droplets with an underlying large-scale coherent motion (see the development of the frames from left column to the right one). It is indispensable that one should view a video of these simulations (Reuteler et al., 1993) in order to fully understand and appreciate the dynamics of this large-scale motion, consisting of constituents with a much finer spatial scale. A gallery of 3-D temperature fields from $R a_{s}=2 \times 10^{6}$ to $4 \times 10^{8}$ for the equilibrium CMB boundary condition can be found in Yuen et al. (1994a).

\subsection{Non-equilibrium results: 2-D finite-differences}

The two-dimensional runs reported in this section have been carried out with the finite-difference method (Honda and Yuen, 1989, 1994) in a Cartesian geometry of aspectratio 5, with $201 \times 1001$ equally distanced grid points. The same depth-dependences in physical properties as in the 3-D model (Honda et al. 1993b) have been em- 


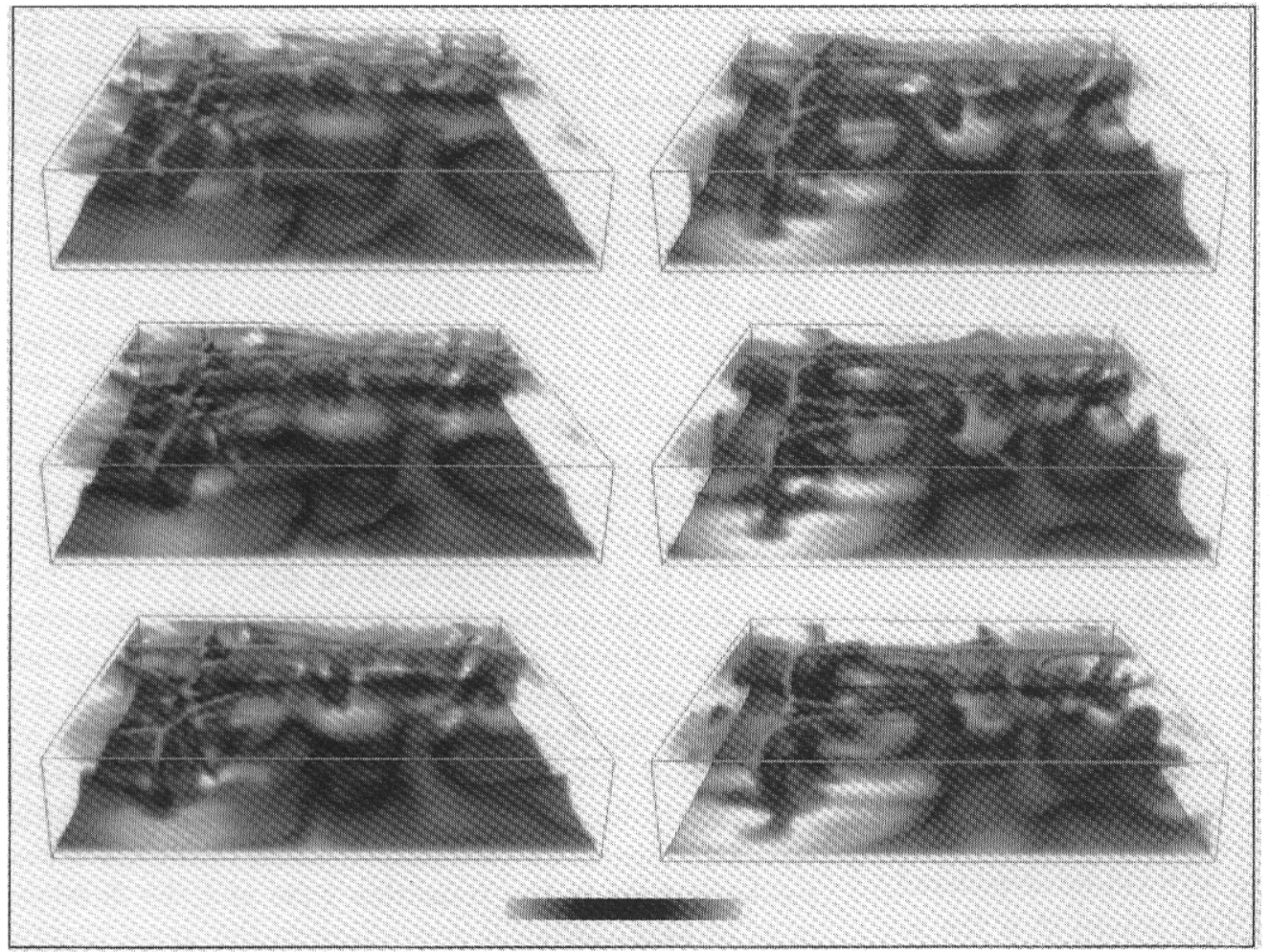

Fig. 2. Sequence of 3-D volumetrically rendered snapshots of temperature. Sequence of events begins with the top left panel, goes down the column and continues along the right column, at equal time intervals. The first and last frames correspond to $t=1.27$ Byrs and 1.59 Byrs. Cold and hot anomalies have been volume rendered in dark and light hues respectively. The void represents temperature values close to average value of 0.5 . The aspect-ratio of the box is $5 \times 5 \times 1$. Surface Rayleigh number is $2 \times 10^{6}$ with an equilibrium (constant temperature) boundary condition at the CMB. Seventy Chebyshev polynomials in the vertical direction and 100 Fourier modes along the horizontal directions are used.

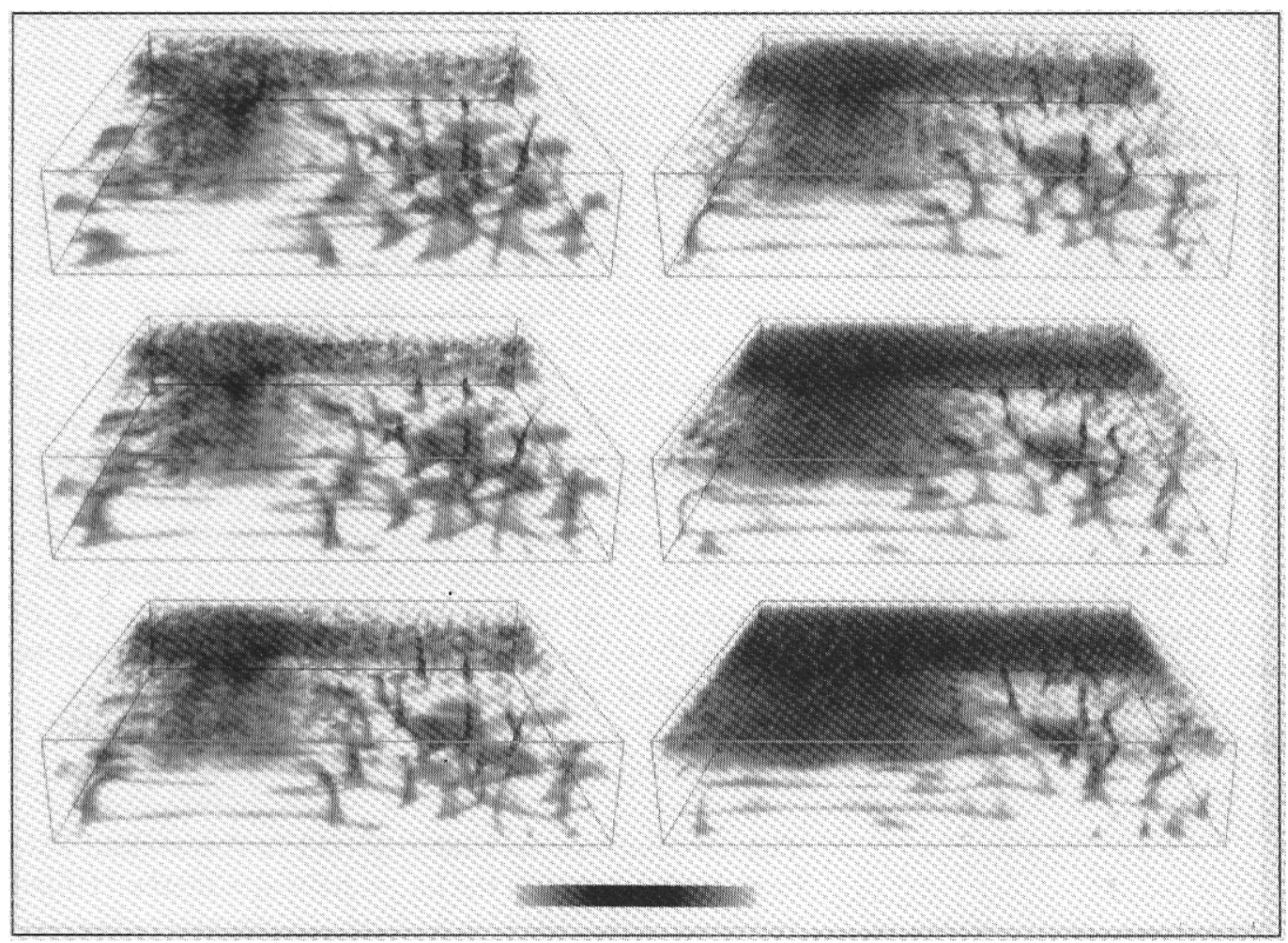

Fig. 3. Sequence of 3-D snapshots for the equilibrium case with $R a_{s}=10^{8}$. Same ordering of frames as in Fig. 2 is employed. Times are $16.7,25.3,35.5,45.7,59.2$ and $71.8 \mathrm{Myr}$. Seventy Chebyshev polynomials in the vertical direction and 180 Fourier modes along the horizontal directions are used in a box of aspect-ratio $5 \times 5 \times 1$. 
ployed. The initial condition used is a fully developed convective state obtained from an equilibrium model at $R a_{s}=10^{8}$. We recognize that the initial conditions of the early periods of the Earth would require a much higher initial $\mathrm{Ra}$ than can be modelled accurately numerically now for both 2-D and 3-D geometries. Rayleigh numbers of $10^{8}$ may correspond to the early Archaean but there are still many uncertainties with such an estimate. Figure 4 shows several snapshots of the 2-D temperature field beginning from the application of the non-equilibrium (core-cooling) boundary conditions. The bottom frame shows the dramatic flush event, which is marked by event E3 in the $R a_{e}$ versus $t$ plot in Fig. 5a. In Fig. 5 a besides showing the evolution of the $R a_{e}$ with time we also show the normalized mass flux at the $670 \mathrm{~km}$ boundary. The normalized mass flux is a ratio between the root mean of the square of the vertical velocity and the root mean of the velocity squared (Steinbach and Yuen, 1992). This quantity is a good indicator of the amount of leakage through the endothermic phase boundary. We recognize E1 as the time when the active hot upwelling first taps the lower-mantle material and E2 as the time when the active cold downwelling first sinks into the lower mantle. E3 is the time of the first catastrophic overturn or flush event. This major event E3 would have taken place some 800 Myrs after the non-equilibrium boundary conditions had been applied. The effective Rayleigh number at this point is around $8 \times 10^{6}$. In Fig. $5 \mathrm{~b}$ we show the $R a_{e}$ and massflux evolution for an aspect-ratio 3 solution, using a fully developed solution at $R a_{o}=10^{8}$ with an equilibrium boundary condition as the starting condition. There is a lengthening of the elapsed time, by about $50 \%$, for similar events to take place in the smaller aspect-ratio configuration. Since the rate of decrease in $R a_{e}$ does not change much with aspect-ratio, the effective Rayleigh number for the major flush event occurred at $R a_{e}$ of $O\left(10^{6}\right)$ (see Fig. 5b). The influences of varying aspectratio on the $R a_{e}$ at which major flush events would occur in non-equilibrium circumstances have important implications on the applications of these Cartesian models to realistic geophysical settings.

\subsection{Non-equilibrium results: Snapshots of 3-D temper- ature fields}

In this section we will display the 3-D results for nonequilibrium forcing driven by time-varying boundary condition as in eqn (2) and by both time-varying boundary condition at $\mathrm{CMB}$ and time-dependent internalheating (eqn (5)) from radioactive decay. The first case considered was started from a fully developed solution based on equilibrium boundary condition at CMB and at $R a_{s}=6 \times 10^{7}$ and no internal heating, taken from a solution in Yuen et al. (1994a). Figure 6 shows a sequence of four snapshots at a time $286 \mathrm{Myr}$ following

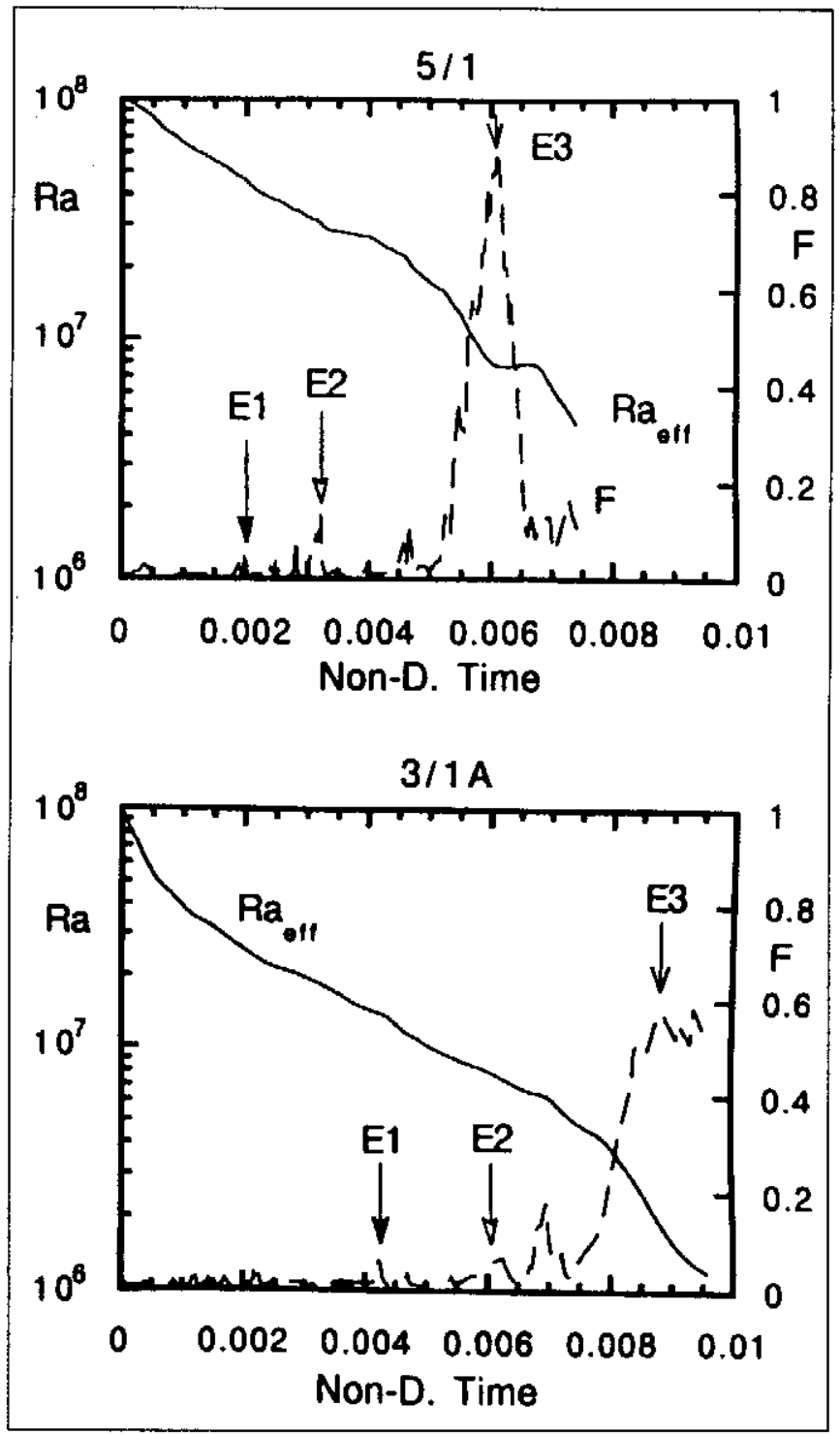

Fig. 5. Temporal evolution of the 2-D mass flux at the $670 \mathrm{~km}$ boundary and of the effective Rayleigh number $R a_{e}$. (a) aspectratio 5 (b) aspect-ratio 3 . Arrows show the approximate time instance at which the different instability events ocuur.

the application of the non-equilibrium boundary condition. Time-sequence goes again from the left to the right column, starting from the upper left-hand corner. The effective Rayleigh numbers for this sequence span from $4 \times 10^{7}$ to $2 \times 10^{7}$. The flush events driven by non-equilibrium conditions display much stronger effect than those developed in equilibrium settings. Prior to the second flush event (upper right-hand corner) we observe percolative structures of the cold material beginning to form (lower left-hand corner). The time interval between the two snapshots is $64 \mathrm{My}$. This observation 


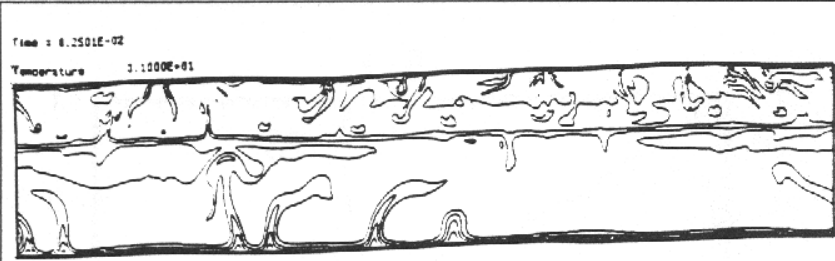

Time $0.2000 \mathrm{E}-02$

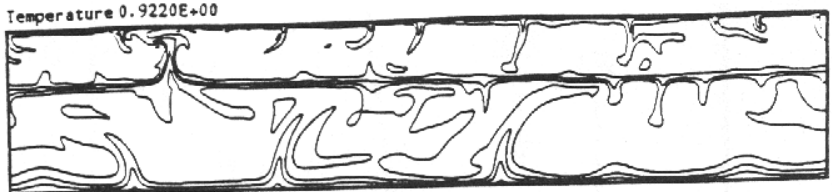

$\mathrm{Ti}=0.3120 \mathrm{E}-02$

Tesper ature $0.8890 \mathrm{E}+00$

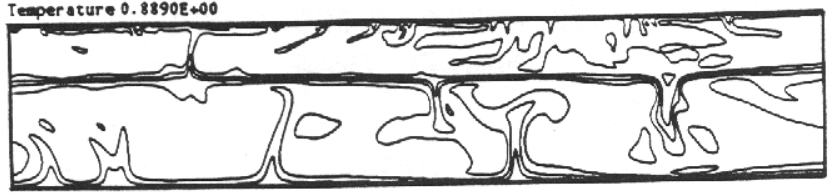

Tíe $=0.6000 E-02$

Teaper ature $0.11635+00$
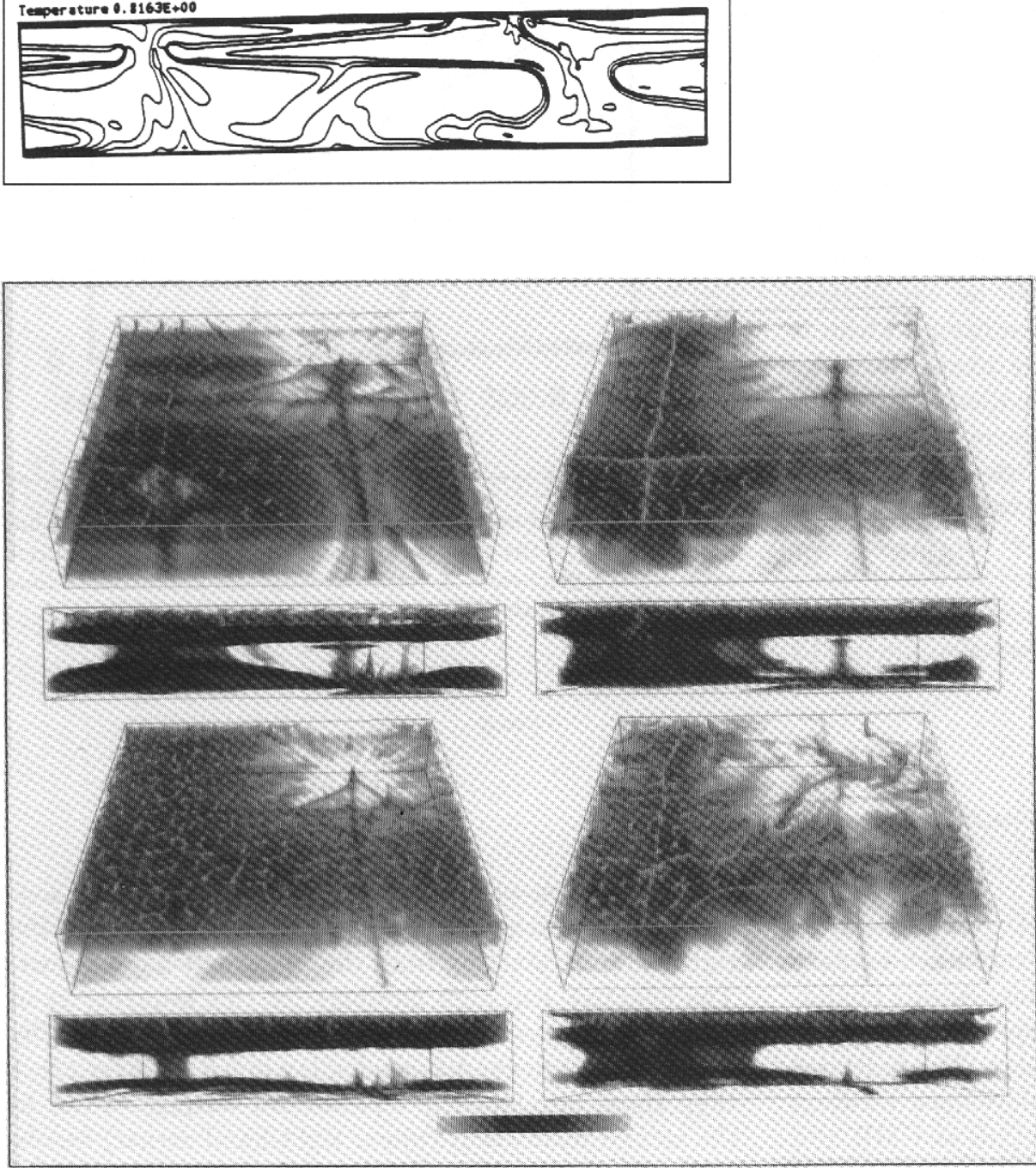

Fig. 4. Snapshots of 2-D temperature fields for non-equilibrium boundary condition. Starting Rayleigh number is $10^{8}$. Contour interval is 0.1 nondimensional temperature. The nondimensional time and the maximum absolute temperature are shown in each panel. $201 \times 1001$ finite-difference grid points with periodic horizontal boundary conditions have been used. To convert to physical time, 0.001 nondimensional time units corresponds to $127 \mathrm{Myr}$.

Fig. 6. Top and side views of the 3-D temperature field generated by a nonequilibrium boundary condition. The same convention in ordering of frames is used as in Fig. 2. Times are 286.13, 393.7, 457.6 and 685.0 Myrs following the application of the non-equilibrium boundary condition. 65-160-160 grid points have been used and the initial Rayleigh number is $6 \times 10^{7}$. 

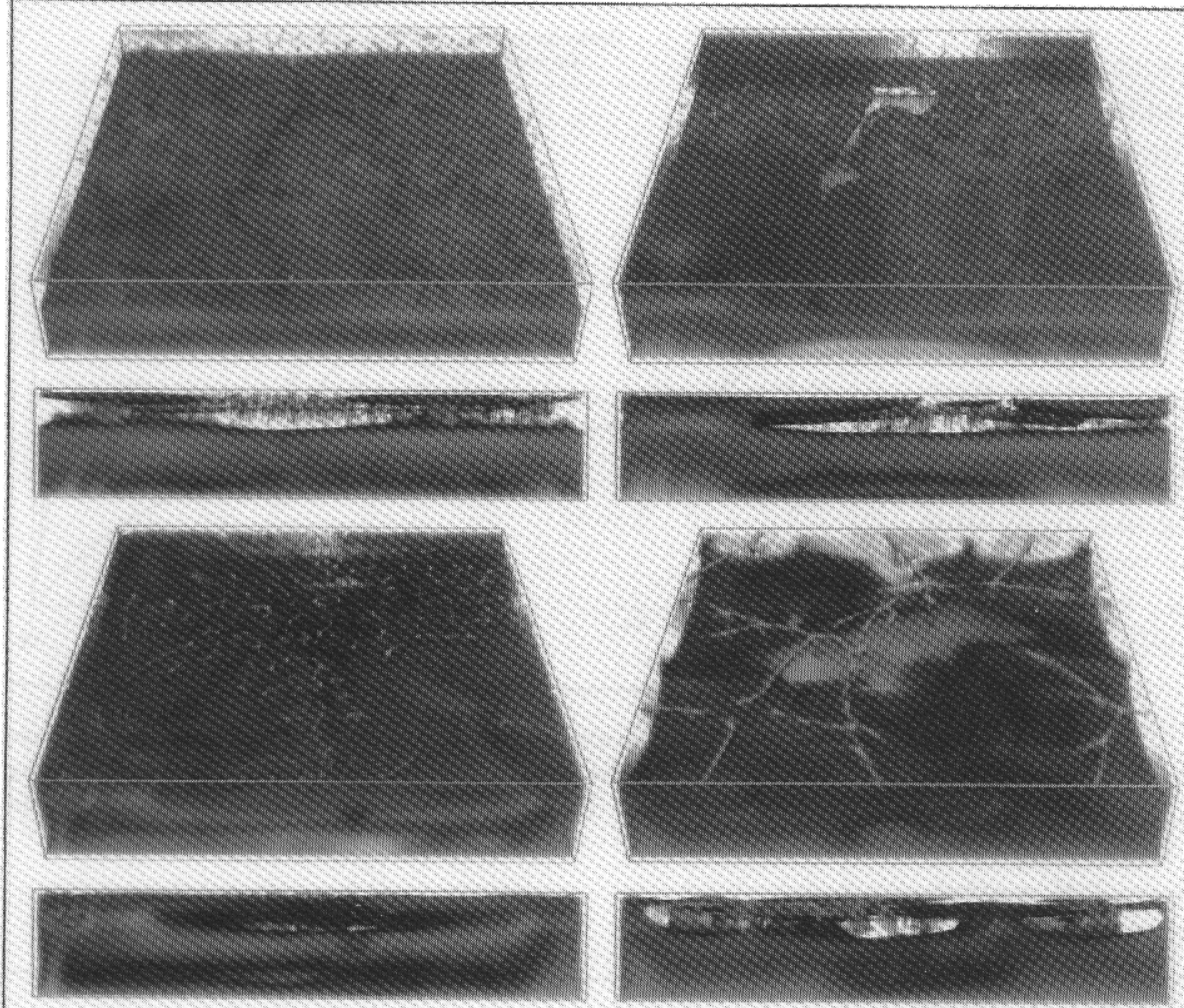

Fig. 7. Top and side views of the temperature field generated by both a non-equilibrium boundary condition and time-dependent internal heating. Same ordering convention is used as in Fig. 2. Times corresponding to the different frames are $1.76,188.8,442.5$ and 1060 Myrs after the onset of the non-equilibrium forcings. The initial Rayleigh number is $2 \times 10^{7}$ and the initial heating strength is 20 with a inverse decay time of 75 . The same number of grid points as in Fig. 6 is used.

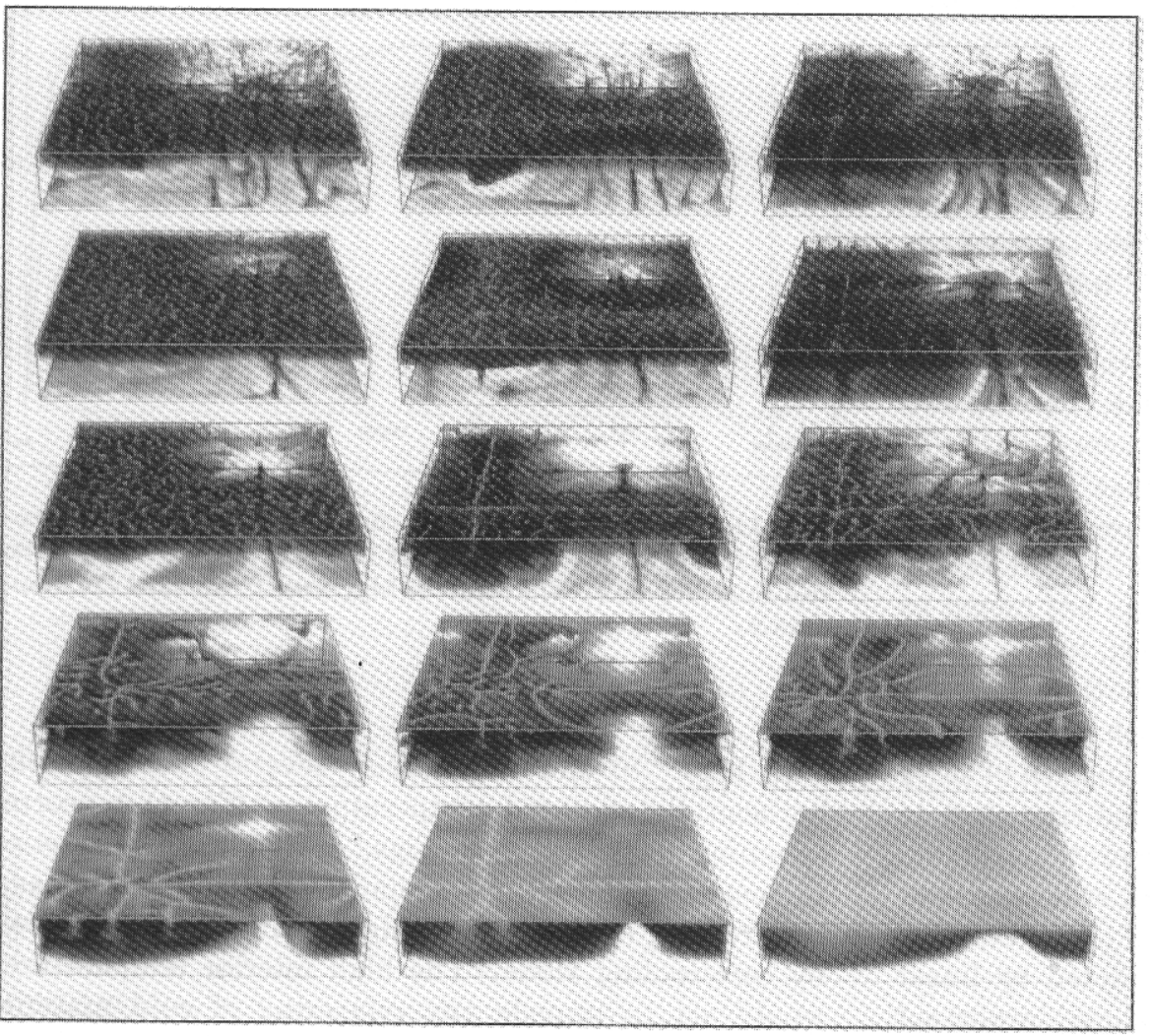

Fig. 8. A series of fifteen 3-D shots corresponding to the entire history of the non-equilibrium run. Sequence starts rowwise from the upper lefthand panel and sweeps right from the top row to the bottom row. The bottom row represents the stage where the convective motions have become extremely sluggish. The initial Rayleigh number is $6 \times 10^{7}$ and non-equilibrium boundary condition is applied at the CMB. Times in Myrs are 1.5, 35.9, $82.8,159.1,222.6,286.1,394.0,457.6$, $684.9,938.9,1192.9,1462.2,2,000.0$, 3605.9 and 6612.9 . 
of a percolative structure forming prior to an instability, in this case the flush event, shows that the flush events driven by non-equilibrium boundary conditions could possibly be regarded as a self-organized critical (SOC) phenomenon (Bak and Chen, 1989; Bak, 1992). Recently Cloetingh et al. (1994) have called attention to SOC-type phenomenon being found in mantle convection because of the presence of threshold phenomena, such as the flush events caused by phase transitions. Figure 7 shows snapshots taken from a case with both non-equilibrium forcing from the CMB boundary condition and time-dependent internal heating, where $R_{0}$ is set to 20 and $\lambda$ is set to 75 , corresponding to a decay time of around 1.7 Byr. The initial condition was taken from a well developed internally heated case, with $R_{0}=20$ and two phase transitions, using the equilibrium boundary condition. The top left-hand frame is taken at a time shortly after the onset of non-equilibrium conditions. For this case of time-dependent internal-healing one could still observe percolative structures on the surface up to the time of the massive flush event (top right-hand panel). We note that the percolative structures may appear differently near the surface because of the strong temperature-dependent rheology, but deeper down such structures may still exist. After the flush event has occurred, a grid-like pattern emerges (lower right-hand panel). The style of flushing between cases with (Fig. 7) and without (Fig. 6) internal heating is quite different in that in the former case there are multiple flush events, while for the time-dependent internally heated case flushing, once started, appears to evolve in more or less continuous fashion without a significantly long period of hiatus.

In Figs 8 . and 9. we show a complete album depicting the evolutionary path of the cases corresponding respectively to Figs 6 and 7 . Inspection of these two figures reveals clearly the transition from a percolative type of structure to a more regular pattern, as the effective Rayleigh number decreases with time. The top two rows of Fig. 8 show especially the precursory nature of the percolative structures prior to a flush event. The time spans covered by Fig. 8 and 9 are respectively 6.6 and 3.8 Byrs. These timescales have come out of the model in a self-consistent manner and are not meant to mimic the Earth's history. The last epoch, for Ra under $10^{6}$, can be regarded as the future. The time scale for evolution is clearly faster for the case with both types of non-equilibrium forcings. The last stage in each of these cases (bottom right frame) reflects a condition close to the conductive state. In fact, convective motions, driven by these non-equilibrium circumstances, still persist at Rayleigh numbers more than an order of magnitude below the critical Rayleigh numbers, predicted by linear stability results. These results would have important implications in the convective state of smaller planetary bodies, such as the Moon, which is generally held to be a dead planetary body today.

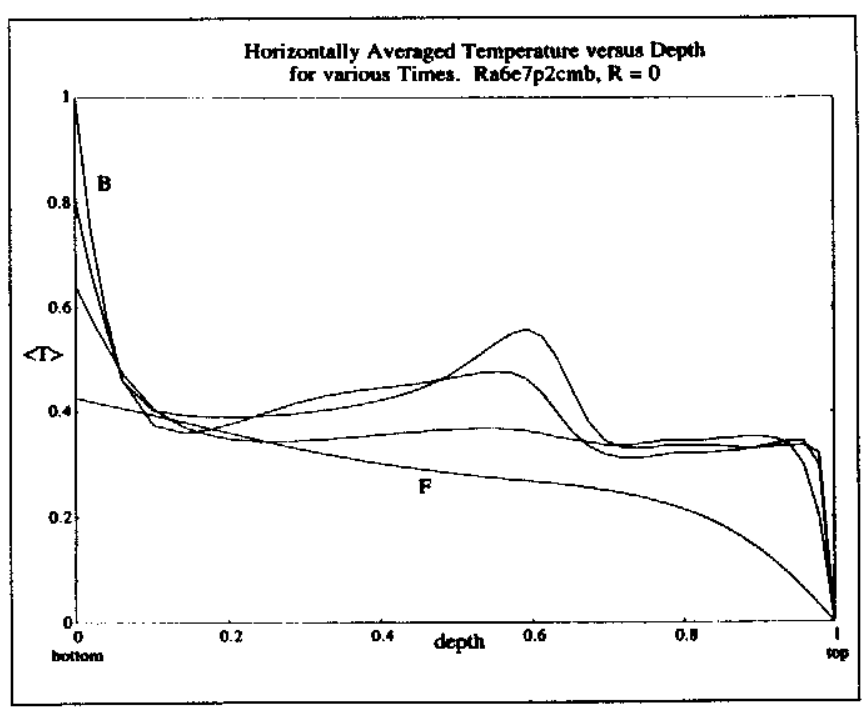

Fig. 10. Horizontally averaged temperature profiles for the case shown in Fig 8 . The curves labled $B$ and $F$ refer to the begining and final instants. The time in $\mathrm{Myr}$ are: 1.52, 394.1, 1192.9 and 6613.3 .

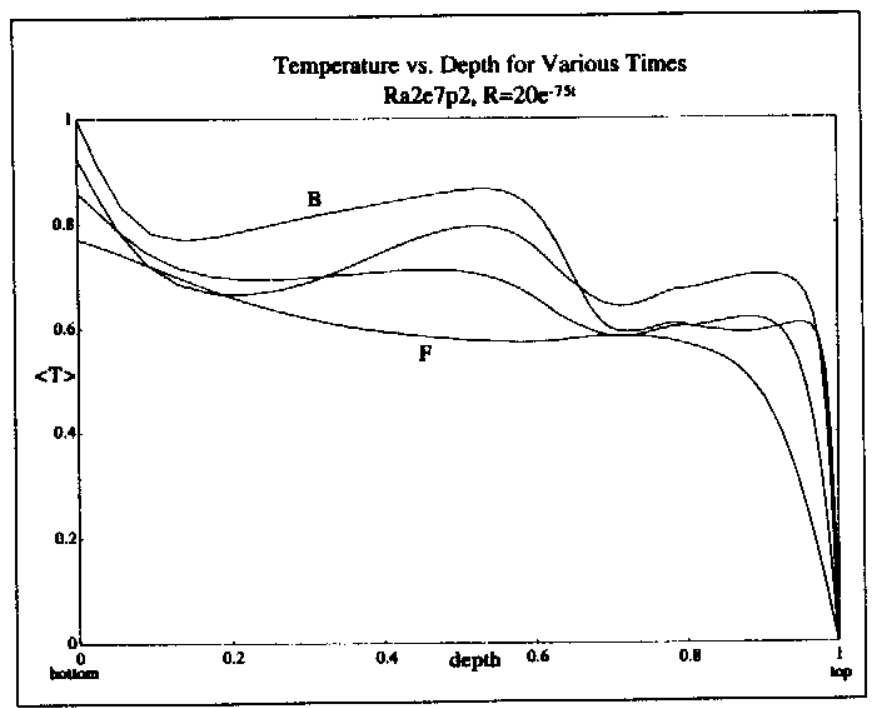

Fig. 11. Horizontally averaged temperature profiles for the case shown in Fig 9. The curves labled $B$ and $F$ refer to the begining and final instants. The time in Myr are: $0,455.2,1474.9$ and 3788.9 .

In Figs. 10 and 11 we show several profiles of the horizontally averaged temperature $\langle T(z, t)\rangle$, corresponding respectively to Figs. 6 and 7 . The interior temperature of the time-dependent internal-heating and core-cooling case are much higher than those produced by core-cooling alone. The high interior temperatures would act to impede any relayering of cold material within the transition zone. In both cases we can discern the evolution of a layered system with a pronounced middle thermal boundary layer to a profile characteris- 


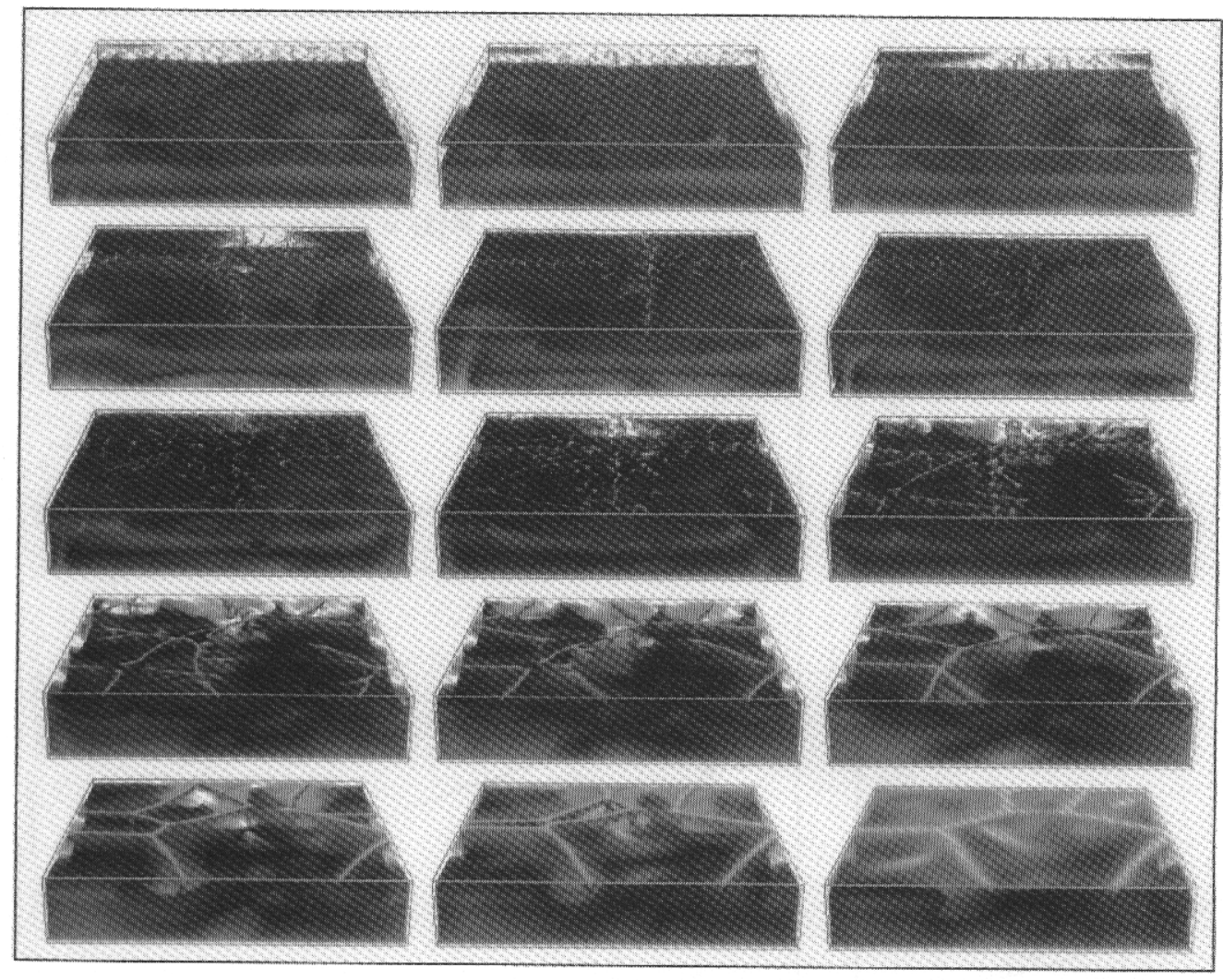

Fig. 9. A series of fifteen 3-D snapshots depicting the entire history of the mantle convection driven by both non-equilibrium boundary condition at $\mathrm{CMB}$ and time-dependent internal heating. The same ordering sequence as in Fig. 8 is used. The initial Rayleigh number is $2 \times 10^{7}$ and the initial internal heating strength is 20 with a dimensionless inverse relaxation time of 75 . Time elapsed (Myr) since non-equilibrium conditions have ensued are: 1.76, 65.1, 128.8, 188.8, 268.0,342,9, 442.5, 593.1, 816.6, 1060.5, 1297.9, 1609.1, 1966.0, 2529.8 and 3780

tic of whole mantle convection. The temperature at the CMB decrease much faster in the purely core-cooling case: $52 \%$ vs $22 \%$ in the case with both core-cooling and time-dependent internal heating. Internal heating obviously plays a very important role on the thermal evolution of the deep mantle.

\subsection{Non-Equilibrium Results: 3-D time-series}

We shall now examine the time-series of the two runs reported in the previous section. Quantities of interest, which would shed light on the impact of nonequilibrium forcings on the thermal evolution of the mantle, would be the variations of the Nusselt and effective Rayleigh numbers with time and the parametric relationships between $N u$ and $R a_{e}$ during the course of the thermal evolution of the coupled core-mantle system. These non-equilibrium solutions go one step beyond the parametrized convection approach (Sharpe and Peltier, 1978; Schubert et al. 1980; Stevenson et al. 1983 ) in that the full set of partial differential equations are employed for describing mantle convection with corecoupling and with the inclusion of the two major phase transitions.
Figure 12 shows $N u(t), R a_{e}(t)$ and $N u$ versus $R a_{e}$ for the non-equilibrium case without any internal heating, whose 3-D snapshots have been shown above in Figs. 6 and 8. Spikes in $N u(t)$ represent the three episodes of flush events, which occurred early in the evolution when $R e_{e}$ was greater than $10^{7}$. The rather fast rate of corecooling delivered by the present core-mantle coupling model is due to the lack of latent and gravitational heat from inner-core formation, which would keep the core hotter and hence slow down the overall cooling process. It is interesting to note that there are two different types of time scales in the cooling process. Initially there is a fast time scale during which convection is strongly time-dependent and flush events take place. After about 1 billion years, when $R a_{e}$ is around $10^{6}$, cooling proceeds at a slower rate. What is important to notice is the non-monotonic character of the cooling curve during the period in which the flush events were occurring. This phenomenon was also found in 2-D simulations (Honda and Yuen, 1994), resulting in a corresponding non-monotonicity in the $N u\left(R a_{e}\right)$ curve. From $R a_{e}=$ $10^{7}$ downwards, the $N u\left(R a_{e}\right)$ relationship has a powerlaw index slightly greater than $1 / 3$, which is higher than what is expected for ordinary Rayleigh-Benard convec- 


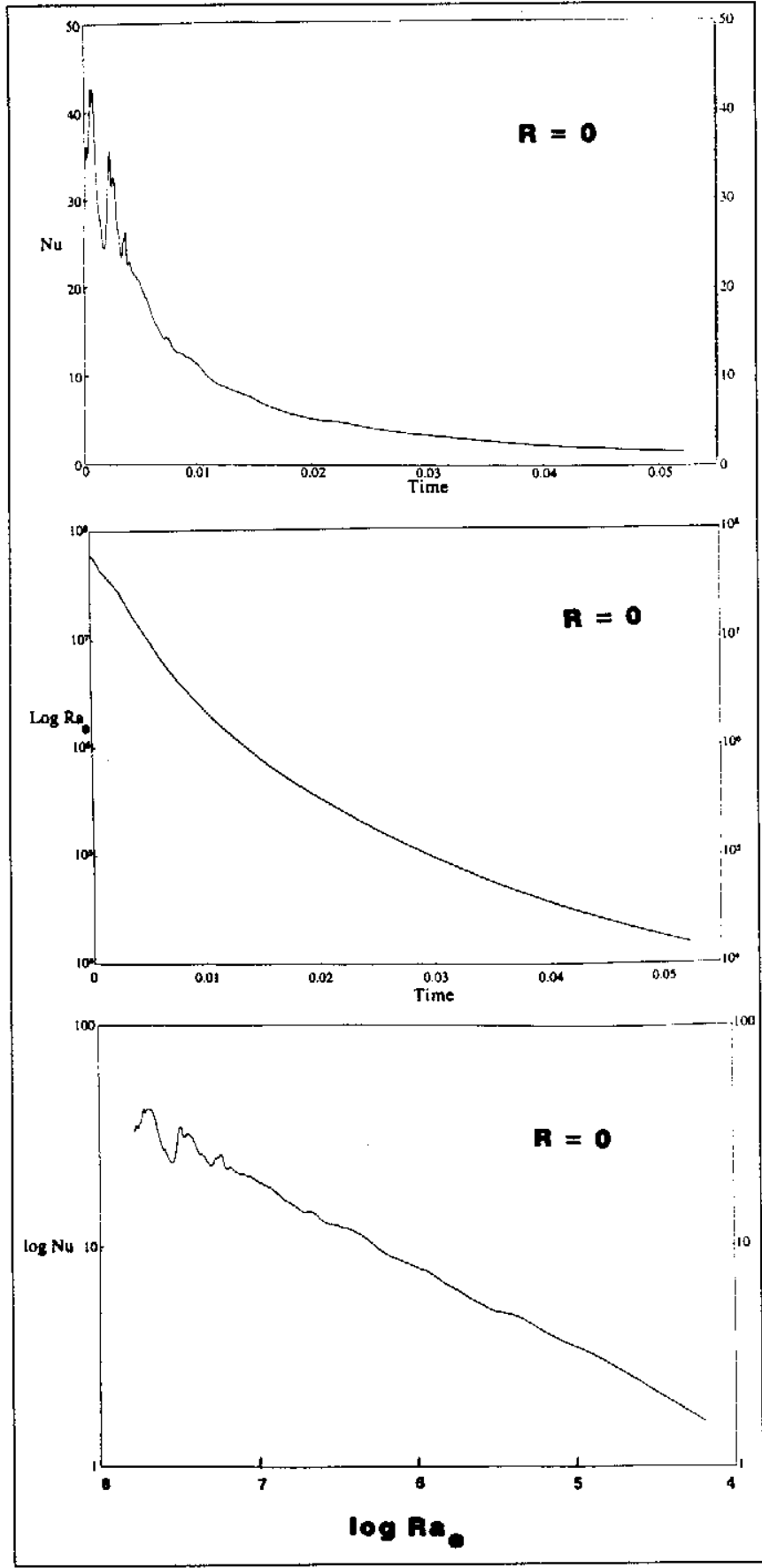

Fig. 12. Time-series for $N u(t), R a_{e}(t)$ and $N u\left(R a_{e}\right)$ for the case corresponding to the snapshots displayed in Fig. 8. For conversion to dimensional time, 0.001 non-dimensional time unit corresponds to $127 \mathrm{Myr}$.

tion under equilibrium conditions in large aspect-ratio configurations (Olson and Corcos, 1980; Hansen and Ebel, 1984).

In Fig, 13 the corresponding $N u(t), R a_{e}(t)$ and $N u$ versus $R a_{e}$ curves for the time-dependent internally heated case, corresponding to Figs. 7 and 9, are displayed. There are three stages in the cooling process as can be observed in the $N u(t), R a_{e}(t)$ and $N u\left(R a_{e}\right)$

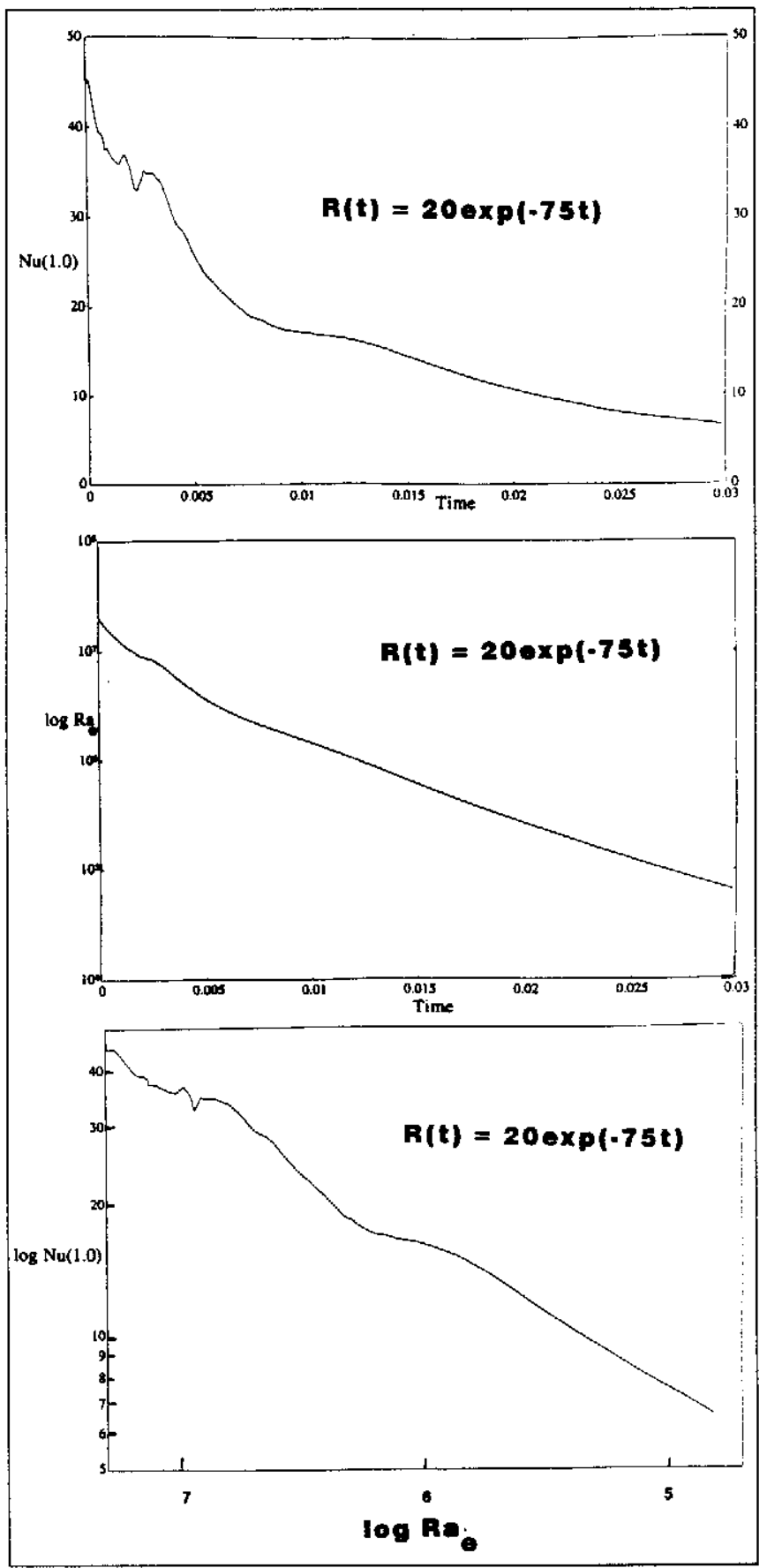

Fig. 13. Time series for $N u(t), R a_{e}(t)$ and $N u\left(R a_{e}\right)$ for the case corresponding to the snapshots displayed in Fig. 9.

curves. First, there is a fast time scale due to the precipitation of the major flush event at around $250 \mathrm{Myr}$ following the application of the non-equilibrium forcings. This is followed by a time scale, which is controlled by the decay time of the internal heating. A slower time scale takes over in the last stage of this evolution, after about 2.5 Byrs. Again the rate of core-cooling is fast because of the simplicity of the thermodynamics of 
the core-cooling model employed. The $N u\left(R a_{e}\right)$ relationship is more complicated than in the case without the time-dependent heating. The power-law index for the dependence of $N u$ on effective Rayleigh number initially is small, because of the high-rate of heat generation. It then increases up to a $R a_{e}$ of around $10^{6}$ and then decreases again. $\Lambda$ fter all of the internal-heating has been exhausted, the power-index increases again. This result points to the more complicated nature of heat-transfer efficiency brought about by both the flush events interacting with time-dependent internal-heating and core-cooling. The common assumption of using a constant power-law dependence in parameterized convection is seen to be violated under non-equilibrium circumstances. When time-depcndent internal heating is present, the issue of $N u\left(R a_{e}\right)$ becomes very complicated because $R a_{e}$ does not explicitly account for the strength of internal heating. This will remain a problem to be resolved in the future.

In Fig. 14 we show the vertical mass fluxes across different depth levels as a function of time for the two cases. The big difference between the two is the greater episodicity of the flush events for the case with the nonequilibrium effect just arising from core-cooling (see Fig. 14 a). This is an extremely important point, since from geological records there are accumulating evidences suggesting the episodic character of singularly large geologic events having taken place in the past (e.g. Larson, 1991, Haggerty, 1994 and Maruyama, 1994). The results presented here point out the distinctly different signature of the flush events caused by the mode of time-dependent internal heating. This finding may in turn be used to place constraints on the amount of internal heating in the mantle throughout the geologic time.

\subsection{Non-equilibrium results: variations of the decay times of internal heating}

In this subsection we will study the influences of varying the decay times of internal heating on non-equilibrium flush events. In the past there has been only one previous work on the influences of time-dependent internal heating in thermal convection without phase changes in a small aspect-ratio box (Daly, 1980). This study was inconclusive because of the simplicity of the model and the low Rayleigh numbers employed at that time. Here we will present results taken from two-dimensional finite-element simulations in a Cartesian box of aspectratio 4 , where the initial Rayleigh number, $R a_{s}$, is set to $10^{7}$. The initial flow field is obtained from a fully developed equilibrium solution at $R a=10^{7}$ and a constant internal heating strength of $K=20$ with two phase transitions. Three different values of the inverse decay time have been studied: 25,75 , and 300 . An inverse decay time, $\lambda$, of 75 would correspond to a dimensional decay time of around $1.7 \mathrm{Byr}$. It is important to employ a wide enough dynamical range, in order to ac-

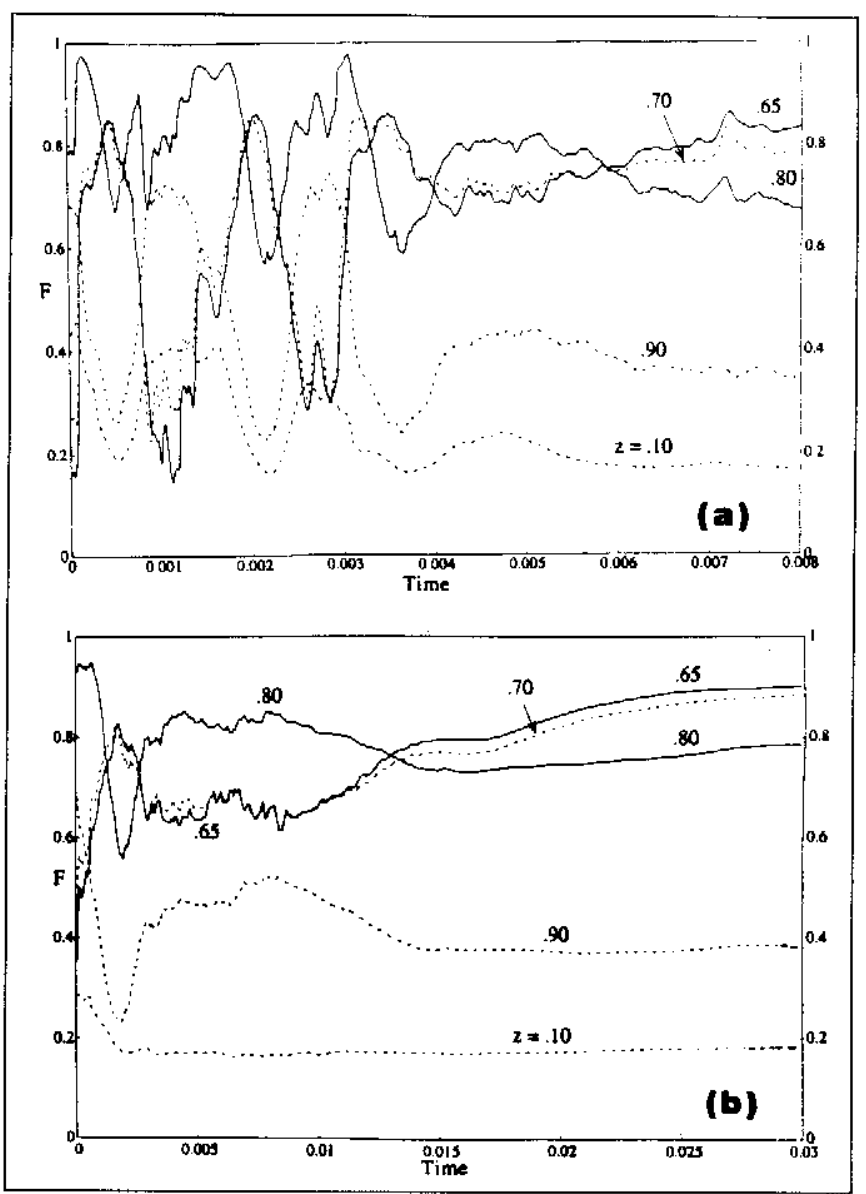

Fig. 14. Vertical mass fluxes $F$ at different depths for the two cases shown in Figs. 8 and 9. Pancls (a) and (b) represent respectively the cases shown in Figs. 8 and 9. The olivine to spinel and the spinel to perovskite transitions are respectively at $z=0.8$ and 0.65 .

commodate the diversities of potential radioactive elements in the mantle. We have used an initial strength of $R_{0}=20$, corresponding to about 1.5 to 2 times chondritic strength. This quantity is a free parameter, which can be increased somewhat without violating seriously geochemical constraints.

In each of thesc cases we will show the average temperature, $\langle T\rangle$, the surface and bottom heat fluxes $\left(F_{1}\right.$ and $F_{0}$ ) as a function of time and the $N u$ versus $R a_{e}$ relationship. In the order of increasing decay times, we show in Figs. 15, 16 and 17 these four quantities respectively for $\lambda=300,75$, and 25 . The faster decay time results in an earlier onset of flush events and induces more subsequent episodes. This result is in accordance with the 3-D findings reported above and sirengthens our argument that the style of flush events in non-equilibrium settings is very much influenced by both the strength and decay-rate of internal-heating. Very fast internalhcating decay rates or no internal heating would enhance the propensity of the mantle to undergo several catastrophic overturns in the course of its thermal history, driven by core-cooling. With slower decay-rates, 


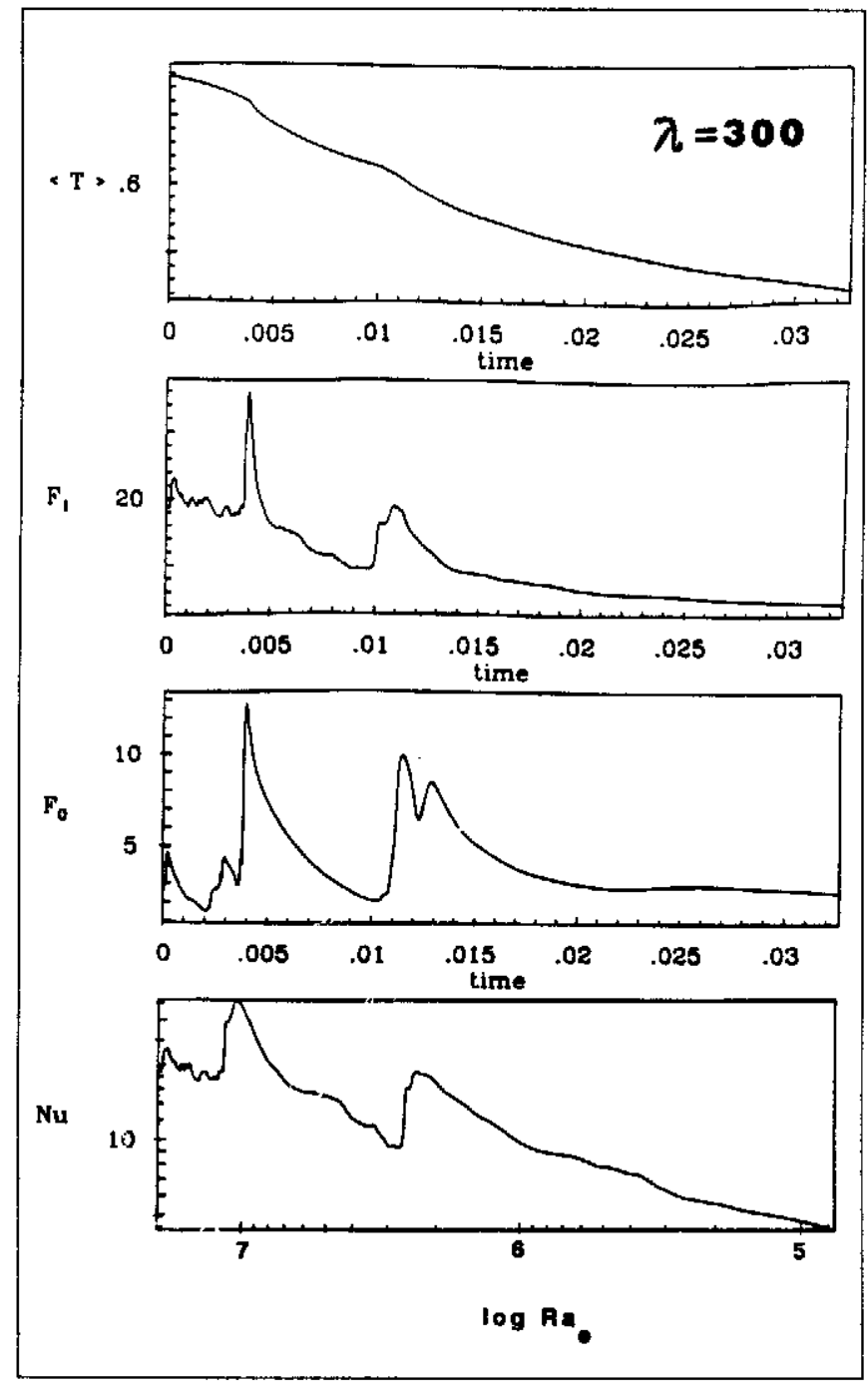

Fig. 15. Temporal evolution of the averaged temperaturc, $\langle T\rangle$, the surface heat flow, $F_{1}$, the heat flow at the CMB, $F_{0}$, and $N u\left(R a_{e}\right)$. This case is taken from two-dimensional finite-element simulations, using $50 \times 200$ elements in a box of aspect-ratio four with reflecting horizontal boundary conditions. The case was started from a initial $R a$ of $10^{7}$. Both non-equilibrium CMB temperature boundary condition and time-dependent internal heating are used with heating dependence of $20 \exp (-300 t)$.

$\lambda=25$, it is even possible for heat to flow back to the core (see $F_{0}(t)$ in Fig. 17). In this case the CMB acts almost like an adiabatic boundary condition with very little cooling of the core, since $F_{0}$ is close to zero. The time series of the surface heat flux changes character, as it goes through the first major flush event. Prior to the flush cvent the time series of $F_{1}(t)$ has many high frequency components. But subsequent to the flush event the heat-flux curve loses its high frequency character. This highly oscillatory temporal phenomenon prior to a major instability is characteristic of self-organized critical event (Bak, 1992) and is complementary to the ear-

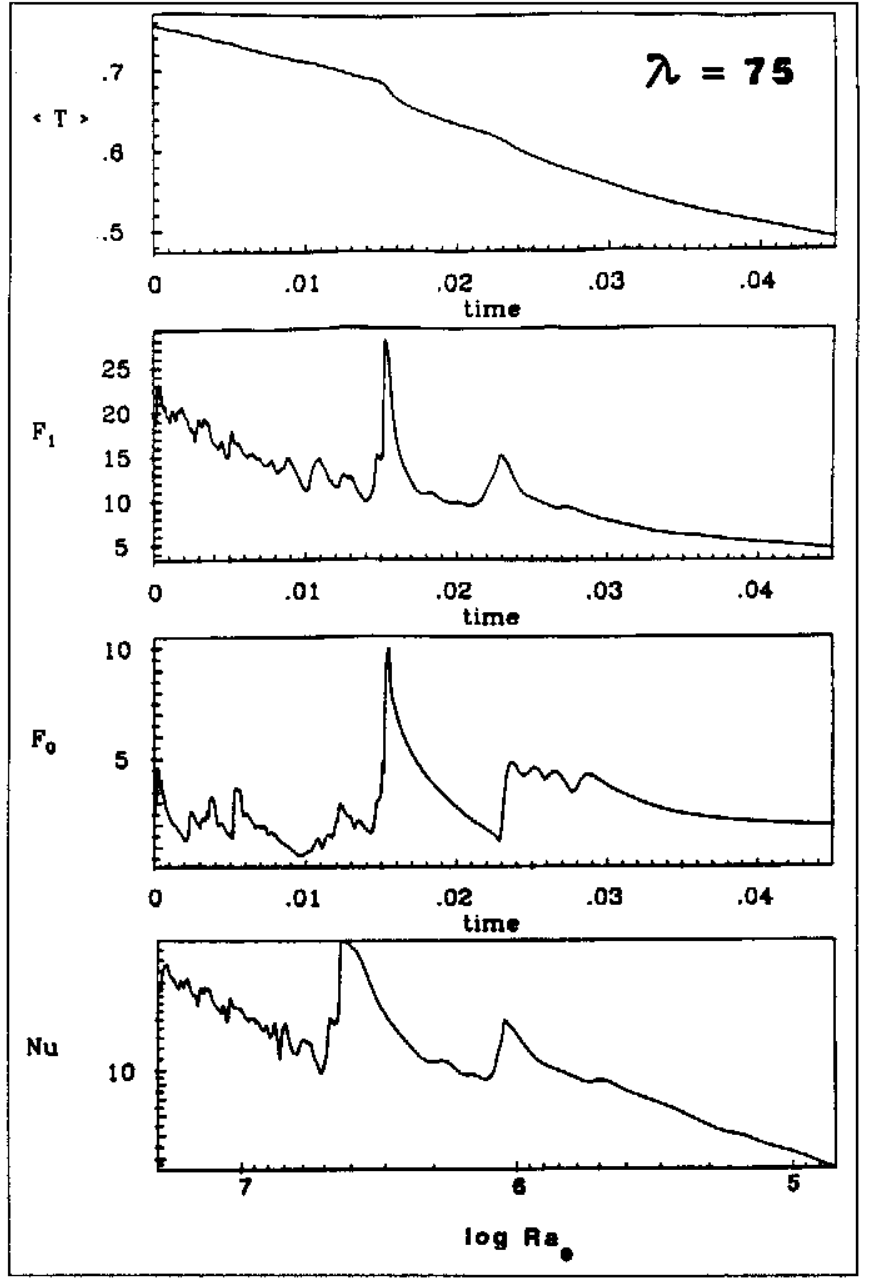

Fig. 16. Same format as in Fig. 13, with the only change being in the heating function of $20 \exp (-75 t)$.

lier observation of the appearance of percolative spatial structures prior to flush events (see in particular Figs. 6 and 8). Cloetingh et al. (1994) have observed a transition in the behavior of the time-series of the sea-level curves during mid-Cretaceous and have attempted to relate this to mantle instability in the Cretaceous (Yuen ct al., 1994b).

In Fig. 18 we have plotted the variation of the powerlaw index as a function of $R a_{e}$ for the three decay times examined above. Very large deviations from the conventionally used power-law exponent value of $1 / 3$ are found. These deviations can have both large positive and negative values, lasting over long periods of time in excess of $\mathrm{O}(100 \mathrm{Myr})$. High values of power-law exponent are induced by the combined effects of time-varying CMB temperature and internal-heating. These results again demonstrate the importance of non-equilibrium effects on the thermal history in the presence of phase transitions (Steinbach et al., 1993). 


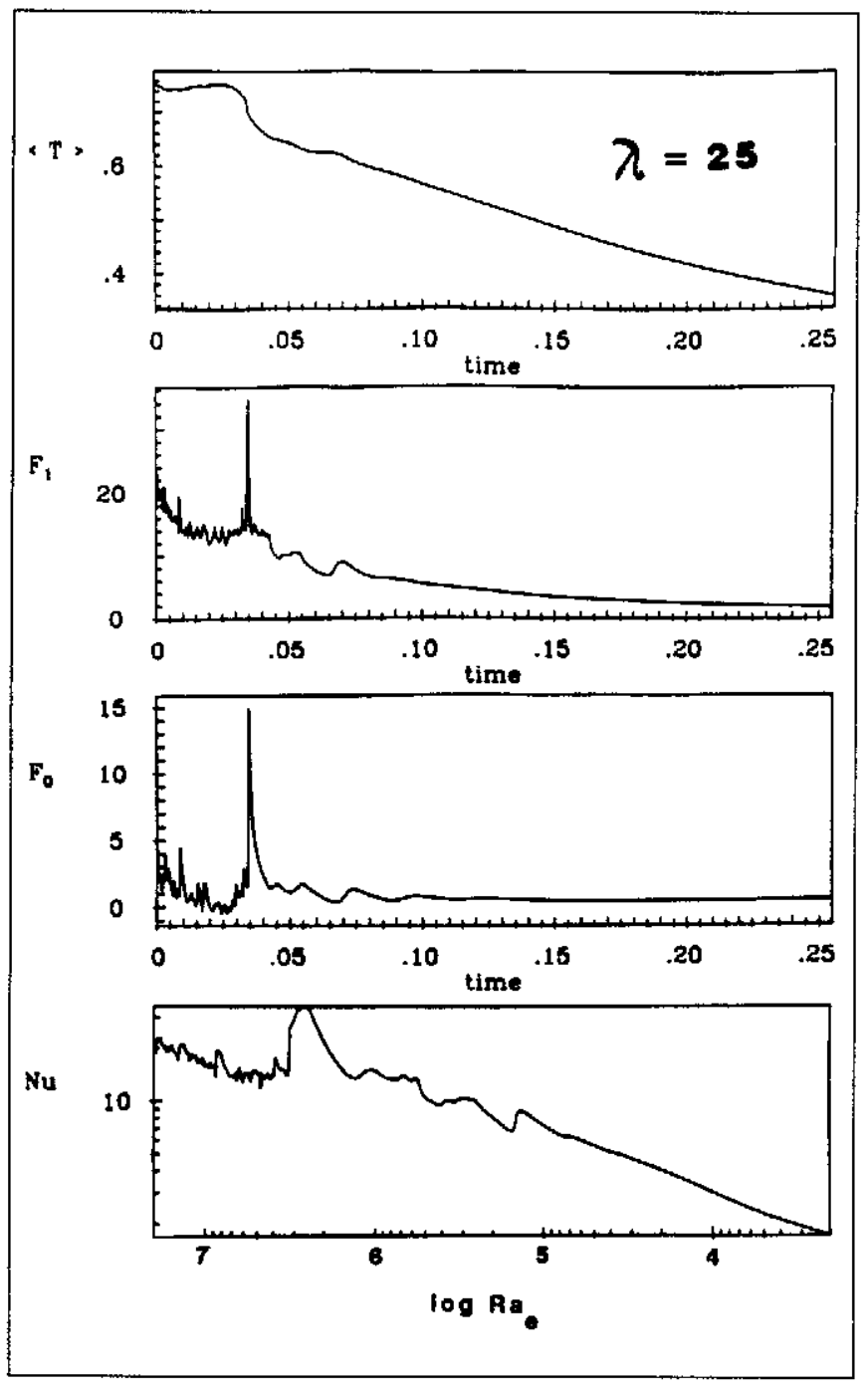

Fig. 17. Same format as in Fig, 13, with the only change being in the heating function of $20 \exp (-25 t)$.

\section{Concluding Remarks}

In this study we have focussed our attention on the several important issues pertaining to the influences of nonequilibrium boundary condition and forcing on mantle flush events. Here we have employed both two and three-dimensional models in order to cover a wide enough parameter space and to obtain complementary results. Both of the 3-D simulations involved extremely long time-integration, as the time-varying Rayleigh number spans several orders of magnitude.

We have found that the effects of non-equilibrium conditions are stronger than those found using the equilibrium (constant) boundary conditions. Non-equilibrium effects produce larger magnitude of the cold anomalies, as well as allow for overshoot of temperature in the hot

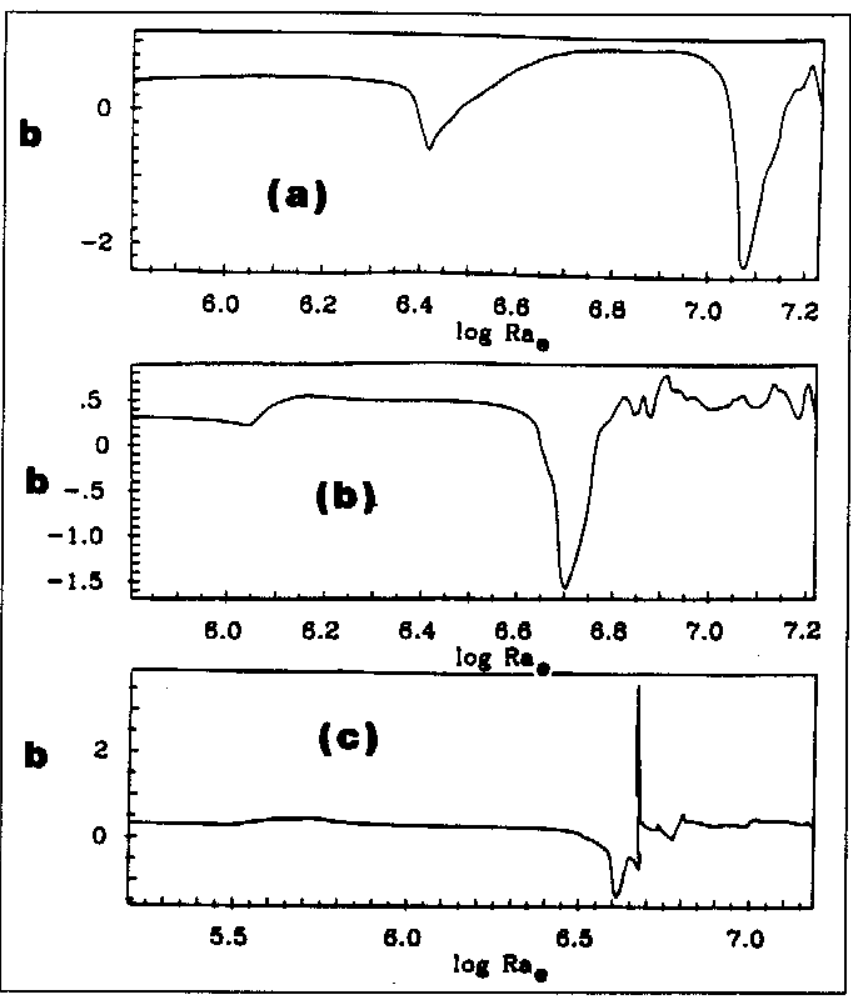

Fig. 18. The time variation of the power-law index, $b$, in the $N u\left(R a_{e}\right)$ relationship. The three panels (a), (b), (c) correspond respectively to the cases presented in Figs. 13, 14, and 15.

plumes, causing melting in the transition zone (Steinbach and Yuen, 1994b). In short, large thermal fluctuations in terms of much colder and hotter anomalies can be produced by the non-equilibrium effects. The large cold and hot anomalies in the deep mantle inferred from seismic tomography and mineral physics (Cadek et al, 1994; Yuen et al., 1993; Yuen et al., 1994b) may indeed reflect the dramatic consequences of mantle convection driven by non-equilibrium forcings.

Mantle flush events in the presence of core-coupling and time-dependent heating are far away from equilibrium condition. Hence the convective system is in general near criticality, i.e. it is ready to develop into an instability without fine tuning of any external control variables. Thus intermittent behavior can develop at high $\mathrm{Ra}$ for core-coupling models in contrast to the flush events for the constant CMB temperature cases, which occur at much lower Rayleigh numbers. As such they may be good examples of self-organized criticality (SOC) manifested in long-term geodynamics. We have shown that there are signs of spatial and temporal manifestations of a SOC state, as shown by the appearance of percolative structure in the cold material prior to the flush event and by the high frequency component in the time series of the heat flow prior to the flush instability. $\Lambda$ dmittedly, the application of this to the real Earth 
would require the use of more realistic rheology in the upper-mantle, such as temperature-dependent and nonlinear creep laws. Nonetheless, it would be useful in the future to analyze the patterns of these spatio-temporal precursors to mantle instabilities in the form of surface topography or gravity anomalies. Clearly there is much more work is needed towards a complcte understanding of this flush phenomenon from the the point of view of SOC, which has proved to be a useful concept in earthquake phenomena (Carlson et al. 1991) and its linkage to plate tectonics (Sornette, 1992). But if this hypothesis holds up, then this mantle instability mechanism is one of the very few examples of SOC drawn from the actual solution of partial differential equations and laws of classical continuum mechanics.

'There is also a major difference in the style of nonequilibrium flush events between models with and without time-dependent internal heating. For core-coupling models, episodic flush events can develop only in the presence of no internal-heating or time-dependent internal heating with a fast enough decay rate. For slower decay rates, flush events become nearly continuous after the initial onset. This signature may allow us to use past geological records for constraining the style of internal heating in the mantle.

We have also found that the cooling curves exhibited by the time-history of $N u$ and $R a_{e}$ do not follow the prediction of steady-state boundary-layer theory, as there are substantial deviations during the time epoch where there are flushing events. Flush events, driven by non-equilibrium conditions, are potentially a very fertile area for research in many respects, both in geophysics and also in nonlinear physics.

Acknowledgements. This research has been supported by National Aeronautics and Space Administration, Division of Mathematical Sciences and Geochemistry programs of the National Science. Foundation and the German D.F.G. We thank Yu.Yu. Podladchikov for very stimulating discussions and Bobby Bolshoi for encouragement. J.J. Smedsmo and G.S. Lauer have becn undergraduate interns of the Minnesota Supercomputer Institute. We also thank the comments from the two anonymous reviewers.

\section{References}

Arkani-Hamed, J., On the thermal cvolution of Venus, J. Geophys. Res.,99, E1, 2019-2033, 1994.

Bak, P., Tang,C. and K. Wiesenfeld, Self-organized criticality, Phys.Rev.A,, 38, 364-374, 1988.

Bak, P. and Chen, K., The physics of fractals, Physica D, 38, 5-12, 1989.

Bak, P., Self-organized criticality in non-conservative models, Physica, A 191, 41-46, 1992.

Balachandar, S., Yuen, D.A., and Reuteler, D., Timedependent three-dimensional compressible convection with depth-dependent properties, Geophys. Res. Lett., 19, 22472250,1992 .

Balachandar, S. and Yuen, D.A., Three-dimensional fully spectral numerical method for mantle convection with depth-dependent properties, J. Comput. Physics, 113, 62-74, 1994.
Balachandar, S., Signature of the transition zone in the tomographic results extracted through the eigenfunctions of the twopoint correlation, in press Geophys. Res. Lett., 1995.

Bayliss, A. and Turkel, E., Mappings and accuracy for Chebyshev pseudo-spectral method, J. Comput. Physics, 91, 174-196, 1992.

Cadck, O., Yuen, D.A., Stcinbach, V., Chopelas, A., and Matyska, C., Lower mantle thermal structure deduced from seismic tomography, mineral physics, and numerical modelling, Earth Planet. Sci. Lett., 121, 385-402, 1994.

Carlson, J.M., Langer, J.S., Shaw, B.E., and 'lang, C., Intrinsic properties of a Burridge-Knopoff model of an earthquake fault, Phys Rev. A, 4/, 881-897, 1991.

Chopelas, A., Thermal properties of beta-Mg2SiO4 at mantle pressures derived from vibrational spectroscopy: implications for the mantle at $400 \mathrm{~km}$ depth, $J$. Geophys. Res., 96, 11817$11829,1991$.

Chopelas, A. and Boehler, R., Thermal expansivity of the lower mantle, Geophys. Res. Lett., 19, 1983-1986, 1992.

Christensen, U.R. and Yuen,D.A., The interaction of a subducting lithospheric slab with a chemical or phase boundary, $J$. Geophys. Res., 89, 4389-4402, 1984.

Christensen, U.R. and Yuen, D.A., Layered convection induced by phase transitions, J. Geophys. Res., 90, 10291-10300, 1985.

Cloetingh, S.A.P.L., Podladchikov, Yu.Yu, Yuen, D.A. and Steinbach, V., Sclf-organized criticality (SOC) as a precursor to flush cvents: Evidences from numerical experiments and sea-level data, E.O.S., Transact. Amer. Geophys. Union, 75, 687, 1994.

Daly, S.F., Convection with decaying heat sources: constant viscosity, Geophys. J. R. Astr. Soc., 61, 519-547, 1980.

Davies, G.F., Thermal histories of convective Earth models and constraints on radiogenic heat production in the Earth, J. Geophys. Res., 85, 2517-2530, 1980.

Fukao, Y., Obayahshi, M. Inoue, H. and Nenbai, M., Subducting slabs stagnant in the transition zone, J. Geophys, Res. 97, 4809$4822,1992$.

Haggerty, S., Superkimberlites: A gcodynamic diamond window to the Earth's core, Earth Planet. Sci. Lett., 122, 57-69, 1994.

Haken, H., Advanced Synergetics, Springer Verlag, New York, 1983.

Hansen, U. and Ebel, A., Experiments with a numerical model related to mantle convection: boundary layer behavior of smalland large-scale flows, Phys. Earth Planet. Inter., 36, 374-390, 1984.

Hansen, U. and Ebel, A., Time-dependent thermal convection - a possible explanation for a multi-scale flow in the Earth's mantle, Geophys. J., 94, 181-191, 1988.

Honda, S. and Yuen, D.A., Mantle convection with moving heatsource anomalies: geophysical and geochemical implications, Earth Planet. Sci. Lett., 96, 349-366, 1989.

Honda, S., Balachandar, S., Yuen, D.A. and Reuteler, D., Threedimensional mantle dynamics with an endothermic phase transition, Geophys. Res. Lett. 20, 221-224, 1993a.

Honda, S., Yuen, D.A., Balachandar, S., and Reuteler, D., Threedimensional instabilitics of mantle convection with multiple phase transitions, Science, 259, 1308-1311, 1993b.

Honda, S. and D.A. Yuen, Cooling model of mantle convection with phase changes: cffects of aspect ratio and initial conditions, J. Phys. Earth., 42, 165-186, 1994.

Ita, J. and King, S.D., Sensitivity of convection with an endothermic phase change to the form of governing equations, initial 
conditions, boundary conditions, and equation of state, J. Gcophys. Res., 99, 15919-15938, 1994.

Ito, E. and Takahashi, E., Postspinel transformations in the system $\mathrm{Mg} 2 \mathrm{SiO} 4-\mathrm{Fe} 2 \mathrm{SiO} 4$ and some geophysical implications, $J$. Geophys, Res., 94, 10637-10,646, 1989.

Katsura, T. and Ito, E., The system Mg2SiO4-Fe2SiO4 at high pressures and temperatures: precise determination of stabilities of olivine, modified spinel and spinel, J. Geophys. Res., 94, 15663-15670, 1989.

I arson, R., Geological consequences of superplumes, Geology, 19, 963-966, 1991.

Leitch, A.M. and Yuen, D.A., Internal heating and thermal constraints on the mantle, Geophys. Res. Lett., 16, 1407-1410, 1989 .

Machetel, P. and Weber, P., Intermittent layered convection in a model mantle with an endothermic phase change at $670 \mathrm{~km}$, Nature, 350, 55-57, 1991.

Maruyama, S., Plume tectonics, J. Geol. Soc. Japan, 100, 24-49, 1994.

Nakakuki, T., Studies of convection in the mantle with the phase and the chemical boundaries by numerical simulations, PII D. Thesis, Ocean Sciences Institute, Univ. 'lokyo, 1993.

Nicolis, G. and I. Prigogine, Self-organization in Non-equilibrium systems, J. Wiley, New York, 1977.

Ohtaki, T. and S. Kaneshima, Continuous high velocity aseismic zone beneath the Izu-Bonin arc, Geophys. Res. Lett., 21, 1-4, 1994.

Olson, P.L. and Corcos, G.M., A boundary layer model for mantle convection with surface plates, Geophys. J. R. Astr. Soc., 62, $195-219,1980$.

Ortoleva, P.J. Geochemical Self-Organization, Oxford Univ. Press, New York, 1994

Reuteler, D.M., Yuen, D.A., Balachandar, S. and Honda, S., Three-dimensional mantle convection: effects of depthdependent properties and multiple phase transitions, Internat. Video J. Eng. Research, 3, 47-62, 1993.

Richter, F.M. and McKenzie, D.P., On some consequences and possible causes of layered mantle convection, J. Geophys. Res., $86,6133-6142,1981$.

Schubert, G., Stevenson, D..J. and Cassen, P., Whole planet cooling and the radiogenic heat source content of the earth and moon, J. Geophys Res., 85, 2531-2538, 1980.

Sharpe, H.N. and Peltier, W.R., Parameterized mantle convection and the Earth's thermal history, Geophys. Res. Lett., 5, 737740,1978 .

Solheim, L.P. and W.R. Peltier, Avalanche effects in phase transition modulated thermal convection: A model of the Earth's mantle, J. Geophys. Ries., 99, 6997-7018, 1994.

Sornette, D., Self-organized criticality, carthquake and platc tectonics, Phys. Rev. Lell., 69, 1287-1290, 1992.

Stauffer, D. and Aharony, A., Introduction to percolation theory, Second edition, Taylor' and Francis, London, 1992.
Stcinbach, V., Hansen, U. and Ebel, A., Compressible convection in the earth's mantle: a comparison of different approaches, Geophys. Res. Lett., 16, 633-635, 1989.

Steimbach, V. and Yuen, D.A., The effects of multiple phase transitions on Venusian mantle convection, Geophys. Res. Lett., 19, 2243-2246, 1992.

Steinbach, V., Yuen, D.A. and Zhao, W., Instabilities from phase transition and the time scales of mantle evolution, Geophys. Res. Lett., 20, 1119-1222, 1993.

Steinbach, V. and Yuen, D.A., Effects of depth-dependent properiies on the thermal anomalies produced in flush instabilities from phase transitions, Phys. Earth Planet. Inter., 86, 165-183, $1994 \mathrm{a}$.

Steinbach, V. and Yuen, D.A., Melting instabilities in the transition zone, Earth Planet Sci. Lett., 127, 67-75, 1994b.

Stevenson, D.J., Spohn, T. and Schubert, G., Magnetism and thermal evolution of the terrestrial planets, Icarus, 54, 466-489, 1983.

Tackley, P.J., Stevenson, D.J., Glatzmaier, G. A. and Schubert, G., Effects of an endothermic phase transition at $670 \mathrm{~km}$ depth in a spherical model of convection in the Earth's mantle, Nature, 361, 699-704, 1993.

Tackley, P. J., Stevenson, D.J., Glatzmaier, G.A. and Schubert, G., Effects of multiple phase transitions in a three-dimensional spherical model of convection in Earth's mantle, J. Geophys. Res., 99, 15, 877-15,902, 1994.

Turcotte, D.L. and Schubert, G., Geodynamics, J. Wiley, New York, 1982.

van der Hilst, R., Èngdahl, R., Spakman,W. and Nolet, G., Tomographic imaging of subducted lithosphere below northwest Pacific island arc, Nature, 353, 37-43, 1991.

van der Hilst, R., Complex morphology of subducted lithosphere in the mantle beneath the Tonga trench, Nature, 374, 154-157, 1995.

Weinstein, S.A., Catastrophic overturn in the Earth's mantle driven by multiple phase changes and internal heat generation, Geophys. Res. Lett., 20, 101-104, 1993.

Yuen, D.A., Cadek, O., Chopelas, A., and Matyska, C., Geophysical inferences of thermal-chemical structures in the lower mantle, Geophys. Rcs. Lett., 20, 889-902, 1993.

Yuen, D.A., Reuteler, D.M., Balachandar, S., Steinbach,V., Malevsky, A.V., and Smcdsmo, J.L., Various influences on three-dimensional mantle convection with phase transitions, Phys. Earlh Planet. Inter, 86, 185-203, 1994a.

Yuen, D.A., Cadek, O.P., Boehler, R., Moser, J. and C.Matyska, Large cold anomalies in the deep mantle and mantle instability in the Cretaceous, Terra Nova, 6, 238-245, 1994b.

Zhao, W., Yuen, D.A, and Honda, S., Multiple phase transitions and the style of mantlc convection, Phys. Earth Planet. Inter., $72,185-210,1992$. 